Research Article

\title{
On the Cohomology and Extensions of $n$-ary Multiplicative Hom-Nambu-Lie Superalgebras
}

\author{
Lijun Tian, ${ }^{1}$ Baoling Guan $\left(\mathbb{D},{ }^{2}\right.$ and Yao $\mathrm{Ma} \mathbb{D}^{3}$ \\ ${ }^{1}$ College of Communications, Qiqihar University, Qiqihar 161006, China \\ ${ }^{2}$ College of Sciences, Qiqihar University, Qiqihar 161006, China \\ ${ }^{3}$ School of Mathematics and Statistics, Northeast Normal University, Changchun 130024, China
}

Correspondence should be addressed to Baoling Guan; 1063686172@qq.com

Received 11 March 2020; Revised 5 May 2020; Accepted 23 May 2020; Published 1 August 2020

Academic Editor: Antonio Scarfone

Copyright () 2020 Lijun Tian et al. This is an open access article distributed under the Creative Commons Attribution License, which permits unrestricted use, distribution, and reproduction in any medium, provided the original work is properly cited.

In this paper, we discuss the representations of $n$-ary multiplicative Hom-Nambu-Lie superalgebras as a generalization of the notion of representations for $n$-ary multiplicative Hom-Nambu-Lie algebras. We also give the cohomology of an $n$-ary multiplicative Hom-Nambu-Lie superalgebra and obtain a relation between extensions of an $n$-ary multiplicative Hom-NambuLie superalgebra $\mathfrak{b}$ by an abelian one $\mathfrak{a}$ and $Z^{1}(\mathfrak{b}, \mathfrak{a})_{\overline{0}}$. We also introduce the notion of $T^{*}$-extensions of $n$-ary multiplicative Hom-Nambu-Lie superalgebras and prove that every finite-dimensional nilpotent metric $n$-ary multiplicative Hom-Nambu-Lie superalgebra over an algebraically closed field of characteristic not 2 in the case $\alpha$ is a surjection is isometric to a suitable $T^{*}$-extension.

\section{Introduction}

In 1996, the concept of $n$-Lie superalgebras was firstly introduced by Daletskii and Kushnirevich in [1]. Moreover, Cantarini and Kac gave a more general concept of $n$-Lie superalgebras again in 2010 in [2]. $n$-Lie superalgebras are more general structures including $n$-Lie algebras ( $n$-ary Nambu-Lie algebras), $n$-ary Nambu-Lie superalgebras, and Lie superalgebras [3].

The general Hom-algebra structures arose first in connection with quasideformation and discretizations of Lie algebras of vector fields. These quasideformations lead to quasi-Lie algebras, a generalized Lie algebra structure in which the skew symmetry and Jacobi conditions are twisted. Hom-Lie algebras, Hom-Lie superalgebras, Hom-Lie bialgebras, Hom-Lie 2-algebras, and quasi-Hom-Lie algebras are discussed in [4-14]. The $n$-ary Hom-Nambu-Lie algebras have been introduced in [15]. It is the generalization of $n$ -ary algebras of Lie type by twisting the identities using linear maps. It includes $n$-ary Hom-algebra structures generalizing the $n$-ary algebras of Lie type such as $n$-ary Nambu algebras, $n$-ary Nambu-Lie algebras, and $n$-ary Lie algebras [16].
Cohomologies are powerful tools in mathematics, which can be applied to algebras and topologies as well as the theory of smooth manifolds or of holomorphic functions. The cohomology of Lie algebras was defined by Chevalley and Eilenberg in order to give an algebraic construction of the cohomology of the underlying topological spaces of compact Lie groups in [17]. The cohomology of Lie superalgebras was introduced by Scheunert and Zhang in [18] and was used in mathematics and theoretical physics: the theory of cobordisms, invariant differential operators, central extensions, and deformations. The theory of cohomology for $n$-ary Hom-Nambu-Lie algebras and $n$-Lie superalgebras can be found in $[19,20]$. This paper generalizes it to $n$-ary multiplicative Hom-Nambu-Lie superalgebras.

The extension is an important way to find a larger algebra and there are many extensions such as general extensions, abelian extensions, nonabelian extensions, double extensions, and Kac-Moody extensions. Abelian extensions and nonabelian extensions of Hom-Lie algebras are, respectively, researched in [21, 22]. The general extensions of $n$-Hom-Lie algebras is researched in [23]. In 1997, Bordemann introduced the notion of $T^{*}$-extensions of Lie algebras in [24]. 
The method of $T^{*}$-extension was used in [25] and was generalized to many other algebras recently in $[26,27]$. This paper researches general extensions and $T^{*}$-extensions of $n$-ary multiplicative Hom-Nambu-Lie superalgebras. In addition, the paper also discusses representations of $n$-ary multiplicative Hom-Nambu-Lie superalgebras as a generalization of the notions of representations for $n$-ary multiplicative Hom-Nambu-Lie algebras.

This paper is organized as follows. In Section 2, we give the representation and the cohomology for an $n$-ary multiplicative Hom-Nambu-Lie superalgebra. In Section 3, we give a one-to-one correspondence between extensions of an $n$-ary multiplicative Hom-Nambu-Lie superalgebras $\mathfrak{b}$ by an abelian one $\mathfrak{a}$ and $Z^{1}(\mathfrak{b}, \mathfrak{a})_{\overline{0}}$. In Section 4 , we introduce the notion of $T^{*}$-extensions of $n$-ary multiplicative Hom-Nambu-Lie superalgebras and prove that every finite-dimensional nilpotent metric $n$-ary multiplicative Hom-Nambu-Lie superalgebra $\left(\mathfrak{g},[\cdot, \cdots, \cdot], \alpha,\langle,\rangle_{\mathfrak{g}}\right)$ over an algebraically closed field of characteristic not 2 such that $\alpha(\mathfrak{g})=\mathfrak{g}$ is isometric to (a nondegenerate ideal of codimension 1 of) a $T^{*}$-extension of a nilpotent $n$-ary multiplicative Hom-Nambu-Lie superalgebra whose nilpotent length is at most a half of the nilpotent length of $\mathfrak{g}$.

\section{2. $n$-ary Hom-Nambu-Lie Superalgebras}

In the paper, let $\mathfrak{g}=\mathfrak{g}_{\overline{0}} \oplus \mathfrak{g}_{\overline{1}}$ be a finite-dimensional $\mathbb{Z}_{2}$-graded vector space. The degree of an element $x$ in $\mathfrak{g}$ will be denoted by $|x|$ and in what follows appearance of $|x|$ will mean that $x$ is a homogeneous element and $|x|$ stands for its degree, where $|x| \in \mathbb{Z}_{2}$ and $\mathbb{Z}_{2}=\{\overline{0}, \overline{1}\}$.

Definition 1. An $n$-ary Hom-Nambu-Lie superalgebra is a triple $\left(\mathfrak{g},[\cdot, \cdots, \cdot],\left\{\alpha_{i}\right\}_{i=1}^{n-1}\right)$ consisting of a $\mathbb{Z}_{2}$-graded vector space $\mathfrak{g}=\mathfrak{g}_{\overline{0}} \oplus \mathfrak{g}_{\overline{1}}$, a multilinear mapping



and a family $\left\{\alpha_{i}\right\}_{i=1}^{n-1}$ of even linear maps $\alpha_{i}: \mathfrak{g} \longrightarrow \mathfrak{g}$, satisfying

$$
\begin{aligned}
& \left|\left[x_{1}, \cdots, x_{n}\right]\right|=\left|x_{1}\right|+\cdots+\left|x_{n}\right| \\
& {\left[x_{1}, \cdots, x_{i}, x_{i+1}, \cdots, x_{n}\right]=-(-1)^{\left|x_{i}\right|\left|x_{i+1}\right|}\left[x_{1}, \cdots, x_{i+1}, x_{i}, \cdots, x_{n}\right],}
\end{aligned}
$$

$$
\begin{gathered}
{\left[\alpha_{1}\left(x_{1}\right), \cdots, \alpha_{n-1}\left(x_{n-1}\right),\left[y_{1}, \cdots, y_{n}\right]\right]=\sum_{i=1}^{n}(-1)^{\left(\left|x_{1}\right|+\cdots+\left|x_{n-1}\right|\right)\left(\left|y_{1}\right|+\cdots+\left|y_{i-1}\right|\right)}} \\
\cdot\left[\alpha_{1}\left(y_{1}\right), \cdots, \alpha_{i-1}\left(y_{i-1}\right),\left[x_{1}, \cdots, x_{n-1}, y_{i}\right], \alpha_{i}\left(y_{i+1}\right), \cdots, \alpha_{n-1}\left(y_{n}\right)\right]
\end{gathered}
$$

where $|x| \in \mathbb{Z}_{2}$ denotes the degree of a homogeneous element $x \in \mathfrak{g}$.
An $n$-ary Hom-Nambu-Lie superalgebra $(\mathfrak{g},[\cdot, \cdots, \cdot]$, $\left.\left\{\alpha_{i}\right\}_{i=1}^{n-1}\right)$ is multiplicative, if $\alpha_{1}=\cdots=\alpha_{n-1}=\alpha$ and the following equality is satisfied:

$$
\alpha\left[x_{1}, \cdots, x_{n}\right]=\left[\alpha\left(x_{1}\right), \cdots, \alpha\left(x_{n}\right)\right], \forall x_{1}, x_{2}, \cdots, x_{n} \in \mathfrak{g} .
$$

Moreover, the multiplicative $n$-ary Hom-Nambu-Lie superalgebra $\left(\mathfrak{g},[\cdot, \cdots, \cdot],\left\{\alpha_{i}\right\}_{i=1}^{n-1}\right)$ is also denoted by $(\mathfrak{g},[\cdot$, $\cdots, \cdot], \alpha)$.

For a multiplicative $n$-ary Hom-Nambu-Lie superalgebra $(\mathfrak{g},[\cdot, \cdots, \cdot], \alpha)$, equation (4) can be read:

$$
\begin{gathered}
{\left[\alpha\left(x_{1}\right), \cdots, \alpha\left(x_{n-1}\right),\left[y_{1}, \cdots, y_{n}\right]\right]} \\
=\sum_{i=1}^{n}(-1)^{\left(\left|x_{1}\right|+\cdots+\left|x_{n-1}\right|\right)\left(\left|y_{1}\right|+\cdots+\left|y_{i-1}\right|\right)} \\
\cdot\left[\alpha\left(y_{1}\right), \cdots, \alpha\left(y_{i-1}\right),\left[x_{1}, \cdots, x_{n-1}, y_{i}\right],\right. \\
\left.\alpha\left(y_{i+1}\right), \cdots, \alpha\left(y_{n}\right)\right] .
\end{gathered}
$$

It is clear that $n$-ary Hom-Nambu-Lie algebras and HomLie superalgebras are particular cases of $n$-ary Hom-NambuLie superalgebras.

Definition 2. Let $\left(\mathfrak{g},[\cdot, \cdots, \cdot],\left\{\alpha_{i}\right\}_{i=1}^{n-1}\right)$ and $\left(\mathfrak{g}^{\prime},[\cdot, \cdots, \cdot]^{\prime}\right.$, $\left.\left\{\alpha^{\prime}{ }_{i}\right\}_{i=1}^{n-1}\right)$ be two $n$-ary Hom-Nambu-Lie superalgebras. A linear map $f: \mathfrak{g} \longrightarrow \mathfrak{g}^{\prime}$ is an $n$-ary Hom-Nambu-Lie superalgebra homomorphism if it satisfies

$$
\begin{aligned}
f\left[x_{1}, \cdots, x_{n}\right] & =\left[f\left(x_{1}\right), \cdots, f\left(x_{n}\right)\right]^{\prime}, \\
f \circ \alpha_{i} & =\alpha_{i}^{\prime} \circ f, \forall i=1, \cdots, n-1 .
\end{aligned}
$$

Example 3. Let $(\mathfrak{g},[\cdot, \cdots, \cdot])$ be an $n$-ary Nambu-Lie superalgebra and let $\rho: \mathfrak{g} \longrightarrow \mathfrak{g}$ be an $n$-ary Nambu-Lie superalgebra endomorphism. Then, $(\mathfrak{g}, \rho \circ[\cdot, \cdots, \cdot], \rho)$ is an $n$-ary multiplicative Hom-Nambu-Lie superalgebra.

Definition 4. Let $\left(\mathfrak{g},[\cdot, \cdots, \cdot]_{\mathfrak{g}},\left\{\alpha_{i}\right\}_{i=1}^{n-1}\right)$ be an $n$-ary HomNambu-Lie superalgebra. A graded subspace $H \subseteq \mathfrak{g}$ is a Hom-subalgebra of $\left(\mathfrak{g},[\cdot, \cdots, \cdot]_{\mathfrak{g}},\left\{\alpha_{i}\right\}_{i=1}^{n-1}\right)$ if $\alpha_{i}(H) \subseteq H$ and $H$ is closed under the bracket operation $[\cdot, \cdots, \cdot]_{\mathfrak{g}}$, i.e., $\left[u_{1}, \cdots, u_{n}\right]_{\mathfrak{g}} \in H$, for all $u_{1}, \cdots, u_{n} \in H$. A graded subspace $H$ $\subseteq \mathfrak{g}$ is a Hom-ideal of $\left(\mathfrak{g},[\cdot, \cdots, \cdot]_{\mathfrak{g}},\left\{\alpha_{i}\right\}_{i=1}^{n-1}\right)$ if $\alpha_{i}(H) \subseteq H$ and $\left[u_{1}, u_{2}, \cdots, u_{n}\right]_{\mathfrak{g}} \in H$, for all $u_{1} \in H$ and $u_{2}, \cdots, u_{n} \in \mathfrak{g}$.

Definition 5. Let $\left(\mathfrak{g},[\cdot, \cdots, \cdot]_{\mathfrak{g}},\left\{\alpha_{i}\right\}_{i=1}^{n-1}\right)$ be an $n$-ary HomNambu-Lie superalgebra. A Hom-ideal $H$ of $\mathfrak{g}$ is abelian if $\left[u_{1}, u_{2}, u_{3}, \cdots, u_{n}\right]_{\mathfrak{g}}=0$ for all $u_{1}, u_{2} \in H$ and $u_{3}, \cdots, u_{n} \in \mathfrak{g}$.

\section{Cohomology for $n$-ary Multiplicative Hom-Nambu-Lie Superalgebras}

Definition 6. Let $(\mathfrak{g},[\cdot, \cdots, \cdot], \alpha)$ be an $n$-ary multiplicative Hom-Nambu-Lie superalgebra. $\mathscr{X}=x_{1} \wedge \cdots \wedge x_{n-1} \in \wedge^{n-1} \mathfrak{g}$ 
is called a fundamental object of $\mathfrak{g}$. For all $z \in \mathfrak{g}, \mathscr{X} \cdot z$ $:=\left[x_{1}, \cdots, x_{n-1}, z\right]$. It is clear that $|\mathscr{X}|=\left|x_{1}\right|+\cdots+\left|x_{n-1}\right|$.

Let $\mathscr{X}=x_{1} \wedge \cdots \wedge x_{n-1}$ and $\mathscr{Y}=y_{1} \wedge \cdots \wedge y_{n-1}$ be two fundamental objects of $\mathfrak{g}$. A bilinear map $[\cdot, \cdot]_{\alpha}: \wedge^{n-1} \mathfrak{g} \times$ $\wedge^{n-1} \mathfrak{g} \longrightarrow \wedge^{n-1} \mathfrak{g}$ defined by

$$
\begin{aligned}
{[\mathscr{X}, \mathscr{Y}]_{\alpha}=} & \sum_{i=1}^{n-1}(-1)^{|\mathscr{X}|\left(\left|y_{1}\right|+\cdots+\left|y_{i-1}\right|\right)} \alpha\left(y_{1}\right) \wedge \cdots \wedge \alpha\left(y_{i-1}\right) \wedge \mathscr{X} \\
& \cdot y_{i} \wedge \alpha\left(y_{i+1}\right) \wedge \cdots \wedge \alpha\left(y_{n-1}\right) .
\end{aligned}
$$

A linear map $\alpha: \wedge^{n-1} \mathfrak{g} \longrightarrow \wedge^{n-1} \mathfrak{g}$ defined by $\alpha(\mathscr{X})=$ $\alpha\left(x_{1}\right) \wedge \cdots \wedge \alpha\left(x_{n-1}\right)$. Then, $\alpha[\mathscr{X}, \mathscr{Y}]_{\alpha}=[\alpha(\mathscr{X}), \alpha(\mathscr{Y})]_{\alpha}$.

Proposition 7 . Let $(\mathfrak{g},[\cdot, \cdots, \cdot], \alpha)$ be an $n$-ary multiplicative Hom-Nambu-Lie superalgebra. Suppose that $\mathscr{X}=x_{1} \wedge \cdots \wedge$ $x_{n-1}, \mathscr{Y}=y_{1} \wedge \cdots \wedge y_{n-1}$ and $\mathscr{Z}=z_{1} \wedge \cdots \wedge z_{n-1}$ are fundamental objects of $\mathfrak{g}$ and $z$ is an arbitrary element in $\mathfrak{g}$. Then,

$$
\alpha(\mathscr{X}) \cdot(\mathscr{Y} \cdot z)=(-1)^{|\mathscr{X}||\mathscr{Y}|} \alpha(\mathscr{Y}) \cdot(\mathscr{X} \cdot z)+[\mathscr{X}, \mathscr{Y}]_{\alpha} \cdot \alpha(z),
$$

$$
[\mathscr{X}, \mathscr{Y}]_{\alpha} \cdot \alpha(z)=-(-1)^{|\mathscr{X}||\mathscr{Y}|}[\mathscr{Y}, \mathscr{X}]_{\alpha} \cdot \alpha(z) \text {. }
$$

Proof. It is easy to see that (9) is equivalent to (6). Using (9), by exchanging $\mathscr{X}$ and $\mathcal{Y}$, we have

$$
\alpha(\mathscr{Y}) \cdot(\mathscr{X} \cdot z)=[\mathscr{Y}, \mathscr{X}]_{\alpha} \cdot \alpha(z)+(-1)^{\mid \mathscr{X} \| \mathscr{Y}} \alpha(\mathscr{X}) \cdot(\mathscr{Y} \cdot z) .
$$

Comparing (9) with (11), we obtain (10).

Definition 8 . Let $(\mathfrak{g},[\cdot, \cdots, \cdot], \alpha)$ be an $n$-ary multiplicative Hom-Nambu-Lie superalgebra and $V=V_{\overline{0}} \oplus V_{\overline{1}}$ be a $\mathbb{Z}_{2^{-}}$ graded vector space over a field $\mathbb{K}$ and $v \in E n d V$. A graded representation $\rho$ of $\mathfrak{g}$ on $V$ is a linear map $\rho: \wedge^{n-1} \mathfrak{g} \longrightarrow$ $\operatorname{End}(V), \mathscr{X} \mapsto \rho(\mathscr{X})=\rho\left(x_{1}, \cdots, x_{n-1}\right)$ such that

$$
\begin{aligned}
& \rho(\mathscr{X})\left(V_{\beta}\right) \subseteq V_{\beta+|\mathscr{X}|}, \quad \forall \beta \in \mathbb{Z}_{2}, \\
& \rho(\alpha(\mathscr{X})) \rho(\mathscr{Y})=(-1)^{|\mathscr{X}||\mathscr{Y}|} \rho(\alpha(\mathscr{Y})) \rho(\mathscr{X})+\rho[\mathscr{X}, \mathscr{Y}]_{\alpha} \circ \nu \\
& \rho\left(\alpha\left(x_{1}\right), \cdots, \alpha\left(x_{n-2}\right),\left[y_{1}, \cdots, y_{n}\right]\right) \circ \nu \\
& =\sum_{i=1}^{n}(-1)^{n-i}(-1)^{\left(\left|x_{1}\right|+\cdots+\left|x_{n-2}\right|\right)\left(\left|y_{1}\right|+\cdots+\left|y_{i}\right| \wedge+\cdots+\left|y_{n}\right|\right)} \\
& \quad \cdot(-1)^{\left|y_{i}\right|\left(\left|y_{i+1}\right|+\cdots+\left|y_{n}\right|\right)} \rho\left(\alpha\left(y_{1}\right), \cdots, \widehat{\alpha\left(y_{i}\right)}, \cdots, \alpha\left(y_{n}\right)\right) \\
& \cdot \rho\left(x_{1}, \cdots, x_{n-2}, y_{i}\right),
\end{aligned}
$$

for $\mathscr{X}, \mathcal{Y} \in \wedge^{n-1} \mathfrak{g}$ and $x_{1}, \cdots, x_{n-2}, y_{1}, \cdots, y_{n} \in \mathfrak{g}$, where the sign $\wedge$ indicates that the element below must be omitted. The $\mathbb{Z}_{2}$-graded representation space $(V, v)$ is said to be a graded $\mathfrak{g}$-module.
We use a supersymmetric notation $\left[x_{1}, \cdots, x_{n-1}, v\right]($ like (3)) to denote $\rho(\mathscr{X}) \cdot v$. Set $\left[x_{1}, \cdots, x_{n-2}, v_{1}, v_{2}\right]=0$ and $(\alpha+v)$ $(x+v)=\alpha(x)+v(v)$ for all $x \in \mathfrak{g}$ and $v \in V$, then $(\mathfrak{g} \oplus V$, $[\cdot, \cdots, \cdot], \alpha+v)$ becomes an $n$-ary multiplicative HomNambu-Lie superalgebra such that $V$ is a $\mathbb{Z}_{2}$-graded abelian ideal of $\mathfrak{g}$, that is,

$$
[V, \underbrace{\mathfrak{g}, \cdots, \mathfrak{g}}_{n-1}] \subseteq V \text { and }[V, V, \underbrace{\mathfrak{g}, \cdots, \mathfrak{g}}_{n-2}]=0 .
$$

In the sequel, we will usually abbreviate $\rho(\mathscr{X}) \cdot v$ with $x \cdot v$.

Example 9. Let $(\mathfrak{g},[\cdot, \cdots, \cdot], \alpha)$ be an $n$-ary multiplicative Hom-Nambu-Lie superalgebra. Then, ad $: \wedge^{n-1} \mathfrak{g} \longrightarrow$ End $(\mathfrak{g}), \mathscr{X} \mapsto \operatorname{ad} \mathscr{X}$ defined by

$$
\operatorname{ad} \mathscr{X}(z)=\mathscr{X} \cdot z
$$

is a graded representation of $\mathfrak{g}$, it is also called the adjoint graded representation of $\mathfrak{g}$.

Definition 10. Let $(\mathfrak{g},[\cdot, \cdots, \cdot], \alpha)$ be an $n$-ary multiplicative Hom-Nambu-Lie superalgebra and $(V, v)$ be a graded $\mathfrak{g}$-module. An $m$-cochain is an $(m+1)$-linear map

$$
f: \underbrace{\wedge^{n-1} \mathfrak{g} \otimes \cdots \otimes \wedge^{n-1} \mathfrak{g}}_{m} \wedge \mathfrak{g} \longrightarrow V
$$

such that

$$
\nu \circ f\left(\mathscr{X}_{1}, \mathscr{X}_{2}, \cdots, \mathscr{X}_{m}, z\right)=f\left(\alpha\left(\mathscr{X}_{1}\right), \alpha\left(\mathscr{X}_{2}\right), \cdots, \alpha\left(\mathscr{X}_{m}\right), \alpha(z)\right)
$$

for all $\mathscr{X}_{1}, \mathscr{X}_{2}, \cdots, \mathscr{X}_{m} \in \wedge^{n-1} \mathfrak{g}$ and $z \in \mathfrak{g}$. We denote the set of $m$-cochain by $C^{m}(\mathfrak{g}, V)$.

Definition 11 . For $m \geq 1$, we define an $m$-coboundary operator $\delta^{m}$ of the $n$-ary multiplicative Hom-Nambu-Lie superalgebra $(\mathfrak{g},[\cdot, \cdots, \cdot], \alpha)$, which is an even linear map, by

$$
\begin{aligned}
& \left(\delta^{m} f\right)\left(\mathscr{X}_{1}, \cdots, \mathscr{X}_{m}, \mathscr{X}_{m+1}, z\right) \\
& =\sum_{i<j}(-1)^{i}(-1)^{\left|X_{i}\right|\left(\left|X_{i+1}\right|+\cdots+\left|X_{j-1}\right|\right)} f \\
& \cdot\left(\alpha\left(\mathscr{X}_{1}\right), \cdots, \widehat{\alpha\left(\mathscr{X}_{i}\right)}, \cdots,\left[\mathscr{X}_{i}, \mathscr{X}_{j}\right]_{\alpha}, \cdots, \alpha\left(\mathscr{X}_{m+1}\right), \alpha(z)\right) \\
& +\sum_{i=1}^{m+1}(-1)^{i}(-1)^{\left|\mathscr{X}_{i}\right|\left(\left|\mathscr{X}_{i+1}\right|+\cdots+\left|X_{m+1}\right|\right)} f \\
& \left.\cdot\left(\alpha\left(\mathscr{X}_{1}\right), \cdots, \alpha \widehat{\mathscr{X}}_{i}\right), \cdots, \alpha\left(\mathscr{X}_{m+1}\right), \mathscr{X}_{i} \cdot z\right) \\
& +\sum_{i=1}^{m+1}(-1)^{i+1}(-1)^{\left|\mathscr{X}_{i}\right|\left(|f|+\left|\mathscr{X}_{1}\right|+\cdots+\left|\mathscr{X}_{i-1}\right|\right)} \alpha^{m}\left(\mathscr{X}_{i}\right)
\end{aligned}
$$




$$
\begin{aligned}
& \cdot f\left(\mathscr{X}_{1}, \cdots, \widehat{X}_{i}, \cdots, \mathscr{X}_{m+1}, z\right) \\
& +(-1)^{m}\left(f\left(\mathscr{X}_{1}, \cdots, \mathscr{X}_{m},\right) \cdot \mathscr{X}_{m+1}\right) \bullet{ }_{\alpha} \alpha^{m}(z),
\end{aligned}
$$

where $\mathscr{X}_{i} \in \wedge^{n-1} \mathfrak{g}, i=1, \cdots, m+1, z \in \mathfrak{g}$ and the last term is defined by

$$
\begin{aligned}
& \left(f\left(\mathscr{X}_{1}, \cdots, \mathscr{X}_{m},\right) \cdot \mathscr{X}_{m+1}\right) \bullet_{\alpha} \alpha^{m}(z) \\
& =\sum_{i=1}^{n-1}(-1)^{\left(|f|+\left|X_{1}\right|+\cdots+\left|X_{m}\right|\right)\left(\left|X_{m+1}^{1}\right|+\cdots+\left|X_{m+1}^{i-1}\right|\right)} \\
& \text { - }\left[\alpha^{m}\left(\mathscr{X}_{m+1}^{1}\right), \cdots, f\left(\mathscr{X}_{1}, \cdots, \mathscr{X}_{m}, \mathscr{X}_{m+1}^{i}\right),\right. \\
& \left.\cdots, \alpha^{m}\left(\mathscr{X}_{m+1}^{n-1}\right), \alpha^{m}(z)\right] \text {, }
\end{aligned}
$$

where $\mathscr{X}_{m+1}=\mathscr{X}_{m+1}^{1} \wedge \cdots \wedge \mathscr{X}_{m+1}^{n-1} \in \wedge^{n-1} \mathfrak{g}$.

Theorem 12. Let $f \in C^{m}(\mathfrak{g}, V)$ be an $m$-cochain. Then, $\delta^{m+1}$ $\circ \delta^{m}(f)=0$.

Proof. See the appendix.

Remark 13. The $m$-coboundary operator $\delta^{m}$ as above is a generalization of the one defined for $n$-ary multiplicative Hom-Nambu-Lie algebras in [16] and for first-class $n$-Lie superalgebras in [20].

The map $f \in C^{m}(\mathfrak{g}, V)$ is called an $m$-supercocycle if $\delta^{m}$ $f=0$. We denote by $Z^{m}(\mathfrak{g}, V)$ the graded subspace spanned by $m$-supercocycles. Since $\delta^{m+1} \circ \delta^{m}(f)=0$ for all $f \in C^{m}(\mathfrak{g}$, $V), \delta^{m-1} C^{m-1}(\mathfrak{g}, V)$ is a graded subspace of $Z^{m}(\mathfrak{g}, V)$. Therefore, we can define a graded cohomology space $H^{m}(\mathfrak{g}, V)$ of $\mathfrak{g}$ as the graded space $Z^{m}(\mathfrak{g}, V) / \delta^{m-1} C^{m-1}(\mathfrak{g}, V)$.

\section{Extensions of $n$-ary Multiplicative Hom-Nambu-Lie Superalgebras}

Definition 14. Let $\left(\mathfrak{g}_{i},[\cdot, \cdots, \cdot]_{i}, \alpha_{i}\right)(i=1,2, \cdots)$ be a family of $n$-ary multiplicative Hom-Nambu-Lie superalgebras over $\mathbb{K}$. $f_{i}: \mathfrak{g}_{i} \rightarrow \mathfrak{g}_{i+1}$ is a morphism of $n$-ary multiplicative Hom-Nambu-Lie superalgebras. The sequence

$$
\mathfrak{g}_{1} \longrightarrow f_{1} \mathfrak{g}_{2} \longrightarrow f_{2} \ldots \longrightarrow \mathfrak{g}_{i} \longrightarrow f_{i} \mathfrak{g}_{i+1} \longrightarrow f^{f_{i+1}} \ldots
$$

is called an exact sequence of $n$-ary multiplicative HomNambu-Lie superalgebras, if it satisfies $\operatorname{ker} f_{i+1}=f_{i}\left(\mathfrak{g}_{i}\right)$ $(i=1,2, \cdots)$.

Definition 15. Let $\left(\mathfrak{g},[\cdot, \cdots, \cdot]_{\mathfrak{g}}, \alpha_{\mathfrak{g}}\right),\left(\mathfrak{a},[\cdot, \cdots, \cdot]_{\mathfrak{a}}, \alpha_{\mathfrak{a}}\right)$ and $(\mathfrak{b}$, $\left.[\cdot, \cdots, \cdot]_{\mathfrak{b}}, \alpha_{\mathfrak{b}}\right)$ be $n$-ary multiplicative Hom-Nambu-Lie superalgebras over $\mathbb{K} . \mathfrak{g}$ is called an extension of $\mathfrak{b}$ by $\mathfrak{a}$ if there is an exact sequence of $n$-ary multiplicative Hom-Nambu-Lie superalgebras:

$$
0 \longrightarrow \mathfrak{a} \longrightarrow \mathfrak{l} \mathfrak{g} \longrightarrow^{\pi} \mathfrak{b} \longrightarrow 0
$$

Let $\left(\mathfrak{g},[\cdot, \cdots, \cdot]_{\mathfrak{g}}, \alpha\right)$ and $\left(\mathfrak{b},[\cdot, \cdots, \cdot]_{\mathfrak{b}}, \beta\right)$ be two $n$-ary multiplicative Hom-Nambu-Lie superalgebras over $\mathbb{K}$. Suppose that $\mathfrak{a}$ is an abelian graded ideal of $\mathfrak{g}$, i.e., $\mathfrak{a}$ is a graded ideal such that

$$
[\mathfrak{a}, \mathfrak{a}, \underbrace{\mathfrak{g}, \cdots, \mathfrak{g}}_{n-2}]=0
$$

We consider the case that $\mathfrak{g i s}$ an extension of $\mathfrak{b}$ by an abelian graded ideal $\mathfrak{a}$ of $\mathfrak{g}$. Let $\tau: \mathfrak{b} \longrightarrow \mathfrak{g}$ be a homogeneous even linear map with $\pi \circ \tau=\mathrm{i} d_{\mathfrak{b}}$ and $\alpha \circ \tau=\tau \circ \beta$. Let $\mathscr{B}=b_{1} \wedge \cdots$ $\wedge b_{n-1} \in \wedge^{n-1} \mathfrak{b}$ and let $\rho: \wedge^{n-1} \mathfrak{b} \longrightarrow \operatorname{End}(\mathfrak{a}), \mathscr{B} \mapsto \tau(\mathscr{B})=$ $\tau\left(b_{1}\right) \wedge \cdots \wedge \tau\left(b_{n-1}\right)$. Then, $\mathfrak{a}$ becomes a graded $\mathfrak{b}$-module. Let us write $\tau(b)=(0, b)$ and then denote the elements of $\mathfrak{g}$ by $(a, b)$ for all $a \in \mathfrak{a}$ and $b \in \mathfrak{b}$. Then, the bracket in $\mathfrak{g}$ is defined by

$$
\begin{aligned}
& {\left[\left(a_{1}, b_{1}\right), \cdots,\left(a_{n}, b_{n}\right)\right]} \\
& \quad=\left(\sum_{i=1}^{n}\left[\tau\left(b_{1}\right), \cdots, a_{i}, \cdots, \tau\left(b_{n}\right)\right]+f\left(\mathscr{B}, b_{n}\right), \mathscr{B} \cdot b_{n}\right),
\end{aligned}
$$

where $f\left(\mathscr{B}, b_{n}\right)=\tau(\mathscr{B}) \cdot \tau\left(b_{n}\right)-\tau\left(\mathscr{B} \cdot b_{n}\right)$ and $\left|\left(a_{i}, b_{i}\right)\right|=$ $\left|a_{i}\right|=\left|b_{i}\right|, \forall 1 \leq i \leq n$. It is easy to see that $f \in C^{1}(\mathfrak{b}, \mathfrak{a})_{\overline{0}}$. Let $\mathscr{A}=a_{1} \wedge \cdots \wedge a_{n-1},(\mathscr{A}, \mathscr{B})=\left(a_{1}, b_{1}\right) \wedge \cdots \wedge\left(a_{n-1}, b_{n-1}\right)$ and $(\alpha$ $(\mathscr{A}), \beta(\mathscr{B}))=\left(\alpha\left(a_{1}\right), \beta\left(b_{1}\right)\right) \wedge \cdots \wedge\left(\alpha\left(a_{n-1}\right), \beta\left(b_{n-1}\right)\right)$. Then,

$$
\begin{aligned}
& (\alpha(\mathscr{A}), \beta(\mathscr{B})) \cdot\left(\left(\mathscr{A}^{\prime}, \mathscr{B}^{\prime}\right) \cdot\left(a_{n}^{\prime}, b_{n}^{\prime}\right)\right)-\sum_{i=1}^{n}(-1)^{|\mathscr{A}|\left(\left|a_{1}{ }^{\prime}\right|+\cdots+\left|a_{i-1}^{\prime}\right|\right)}\left[\left(\alpha\left(a_{1}^{\prime}\right),\right.\right. \\
& \left.\left.\beta\left(b_{1}^{\prime}\right)\right), \cdots,(\mathscr{A}, \mathscr{B}) \cdot\left(a_{i}^{\prime}, b_{i}^{\prime}\right), \cdots,\left(\alpha\left(a_{n}^{\prime}\right), \beta\left(b_{n}^{\prime}\right)\right)\right] \\
& =(\alpha(\mathscr{A}), \beta(\mathscr{B})) \cdot\left(\sum_{i=1}^{n}\left[\tau\left(b_{1}^{\prime}\right), \cdots, a_{i}^{\prime}, \cdots, \tau\left(b_{n}^{\prime}\right)\right]+f\left(\mathscr{B}^{\prime}, b_{n}^{\prime}\right), \mathscr{B}^{\prime} \cdot b_{n}^{\prime}\right) \\
& -\sum_{i=1}^{n}(-1)^{|\mathscr{Q}|\left(\left|a_{1}{ }^{\prime}\right|+\cdots+\left|a_{i-1}^{\prime}\right|\right)} \cdot\left[\left(\alpha\left(a_{1}^{\prime}\right), \beta\left(b_{1}^{\prime}\right)\right), \cdots,\right. \\
& \left(\left(\sum_{j=1}^{n-1}\left[\tau\left(b_{1}\right), \cdots, a_{j}, \cdots, \tau\left(b_{n-1}\right), \tau\left(b_{i}^{\prime}\right)\right]+\tau(\mathscr{B}) \cdot a_{i}^{\prime}\right.\right. \\
& \left.\left.\left.+f\left(\mathscr{B}, b_{i}^{\prime}\right)\right), \mathscr{B} \cdot b_{i}^{\prime}\right), \cdots,\left(\alpha\left(a_{n}^{\prime}\right), \beta\left(b_{n}^{\prime}\right)\right)\right] \\
& =\left(\left(\tau(\beta(\mathscr{B})) \cdot\left(\sum_{i=1}^{n}\left[\tau\left(b_{1}^{\prime}\right), \cdots, a_{i}^{\prime}, \cdots, \tau\left(b_{n}^{\prime}\right)\right]\right)\right.\right. \\
& +\sum_{j=1}^{n-1}\left[\tau\left(\beta\left(b_{1}\right)\right), \cdots, \alpha\left(a_{j}\right), \cdots, \tau\left(\beta\left(b_{n-1}\right)\right), \tau\left(\mathscr{B}^{\prime} \cdot b_{n}^{\prime}\right)\right] \\
& \left.\left.+\tau(\beta(\mathscr{B})) \cdot f\left(\mathscr{B}^{\prime}, b_{n}^{\prime}\right)+f\left(\beta(\mathscr{B}), \mathscr{B}^{\prime} \cdot b_{n}^{\prime}\right)\right), \beta(\mathscr{B}) \cdot\left(\mathscr{B}^{\prime} \cdot b_{n}\right)\right) \\
& -\sum_{i=1}^{n}(-1)^{|\mathscr{A}|\left(\left|a_{1}{ }^{\prime}\right|+\cdots+\left|a_{i-1}^{\prime}\right|\right)} \cdot\left(\left(\sum _ { j = 1 } ^ { n - 1 } \left[\tau\left(\beta\left(b_{1}^{\prime}\right)\right), \cdots,\right.\right.\right. \\
& \left.\left[\tau\left(b_{1}\right), \cdots, a_{j}, \cdots, \tau\left(b_{n-1}\right), \tau\left(b_{i}^{\prime}\right)\right], \cdots, \tau\left(\beta\left(b_{n}^{\prime}\right)\right)\right] \\
& +\left[\tau\left(\beta\left(b_{1}^{\prime}\right)\right), \cdots, \tau(\mathscr{B}) \cdot a_{i}^{\prime}, \cdots, \tau\left(\beta\left(b_{n}^{\prime}\right)\right)\right] \\
& +\left[\tau\left(\beta\left(b_{1}^{\prime}\right)\right), \cdots, f\left(\mathscr{B}, b_{i}^{\prime}\right), \cdots, \tau\left(\beta\left(b_{n}^{\prime}\right)\right)\right] \\
& +\sum_{j \neq i}\left[\tau\left(\beta\left(b_{1}^{\prime}\right)\right), \cdots, \alpha\left(a_{j}^{\prime}\right), \cdots, \tau\left(\mathscr{B} \cdot b_{i}^{\prime}\right), \cdots, \tau\left(\beta\left(b_{n}^{\prime}\right)\right)\right] \\
& \left.\left.+f\left(\beta\left(b_{1}^{\prime}\right), \cdots, \mathscr{B} \cdot b_{i}^{\prime}, \cdots, \beta\left(b_{n}^{\prime}\right)\right)\right),\left[\beta\left(b_{1}^{\prime}\right), \cdots, \mathscr{B} \cdot b_{i}^{\prime}, \cdots, \beta\left(b_{n}^{\prime}\right)\right]\right) \\
& =\left(\delta^{1} f\left(\mathscr{B}, \mathscr{B}^{\prime}, b_{n}^{\prime}\right), 0\right) \text {. }
\end{aligned}
$$


Therefore, $f \in Z^{1}(\mathfrak{b}, \mathfrak{a})_{\overline{0}}$.

Conversely, suppose that an abelian $n$-ary multiplicative Hom-Nambu-Lie superalgebras $\mathfrak{a}$ is a graded $\mathfrak{b}$-module, $\rho$ $(\mathscr{B}) \cdot a:=\tau(\mathscr{B}) \cdot a$, and $f \in Z^{1}(\mathfrak{b}, \mathfrak{a})_{\overline{0}}$. Let $\mathfrak{g}:=(\mathfrak{a}, \mathfrak{b})=\{(x, y)$ $\mid x \in \mathfrak{a}, y \in \mathfrak{b}\}, \alpha^{\prime}:=\alpha+\beta$, where $(\alpha+\beta)(x, y)=(\alpha(x), \beta(y))$, $x \in \mathfrak{a}, y \in \mathfrak{b}$. Then $\left(\mathfrak{g}, \alpha^{\prime}\right)$ is an $n$-ary multiplicative HomNambu-Lie superalgebra with the bracket defined by (24). Then we can define an exact sequence

$$
0 \longrightarrow \mathfrak{a} \longrightarrow^{l} \mathfrak{g} \longrightarrow{ }^{\pi} \mathfrak{b} \longrightarrow 0
$$

where $\iota(a)=(a, 0), \pi(a, b)=b$. Thus $\mathfrak{g}$ is an extension of $\mathfrak{b}$ by $\mathfrak{a}$ and $\iota(\mathfrak{a})$ is an abelian graded ideal of $\mathfrak{g}$.

Therefore, we get the following theorem.

Theorem 16. Suppose that $\left(\mathfrak{a},[\cdot, \cdots, \cdot]_{\mathfrak{a}}, \alpha\right)$ and $\left(\mathfrak{b},[\cdot, \cdots, \cdot]_{\mathfrak{b}}\right.$, $\beta)$ are two n-ary multiplicative Hom-Nambu-Lie superalgebras over $\mathbb{K}$ and $\mathfrak{a}$ is abelian. Then, there is a one-to-one correspondence between extensions of $\mathfrak{b}$ by $\mathfrak{a}$ and $Z^{1}(\mathfrak{b}, \mathfrak{a})_{\overline{0}}$.

\section{5. $T^{*}$-Extensions of $n$-ary Multiplicative Hom-Nambu-Lie Superalgebras}

Let $(\mathfrak{g},[\cdot, \cdots, \cdot], \alpha)$ be an $n$-ary multiplicative Hom-NambuLie superalgebra and $\mathfrak{g}^{*}$ be its dual space. Since $\mathfrak{g}=\mathfrak{g}_{\overline{0}} \oplus \mathfrak{g}_{\overline{1}}$ and $\mathfrak{g}^{*}=\mathfrak{g}_{0}^{*} \oplus \mathfrak{g}_{1}^{*}$ are $\mathbb{Z}_{2}$-graded vector space, the direct sum $\mathfrak{g} \oplus \mathfrak{g}^{*}=\left(\mathfrak{g}_{\overline{0}} \oplus \mathfrak{g}_{0}^{*}\right) \oplus\left(\mathfrak{g}_{\overline{1}} \oplus \mathfrak{g}_{1}^{*}\right)$ is a $\mathbb{Z}_{2}$-graded vector space. In the sequel, whenever $x+f \in \mathfrak{g} \oplus \mathfrak{g}^{*}$ appears, it means that $x+f$ is homogeneous and $|x+f|=|x|=|f|$.

Lemma 17. Let $\mathfrak{g}^{*}$ be the dual $\mathbb{Z}_{2}$-graded vector space of an $n$-ary multiplicative Hom-Nambu-Lie superalgebra ( $\mathfrak{g}$, $[\cdot, \cdots, \cdot], \alpha)$. Let us consider the even linear map $a d^{*}$ $: \wedge^{n-1} \mathfrak{g} \longrightarrow \operatorname{End}\left(\mathfrak{g}^{*}\right)$ defined by

$$
a d^{*}(\mathscr{X})(f)(z)=-(-1)^{|\mathscr{X}||f|} f(\operatorname{ad} \mathscr{X}(z)),
$$

for all $\mathscr{X} \in \wedge^{n-1} \mathfrak{g}, f \in \mathfrak{g}^{*}$ and $z \in \mathfrak{g}$. Then, ad $d^{*}$ is a representation of $\mathfrak{g}$ on $\mathfrak{g}^{*}$ if and only if the following conditions hold:

$$
\begin{aligned}
\operatorname{ad}(\mathscr{X}) \operatorname{ad} \alpha(\mathscr{Y})-(-1)^{|\mathscr{X}||\mathscr{Y}|} \operatorname{ad}(\mathscr{Y}) \operatorname{ad} \alpha(\mathscr{X})=\alpha \circ \operatorname{ad}[\mathscr{X}, \mathscr{Y}]_{\alpha} ; \\
\operatorname{ad}\left(x_{1}, \cdots, x_{n-2}, y_{i}\right) \operatorname{ad}\left(\alpha\left(y_{1}\right), \cdots, \widehat{\alpha\left(y_{i}\right)}, \cdots, \alpha\left(y_{n}\right)\right) \\
=(-1)^{\left(\left|x_{1}\right|+\cdots+\left|x_{n-2}\right|\right)\left(\left|y_{1}\right|+\cdots+\left|y_{i}\right| \wedge+\cdots+\left|y_{n}\right|\right)} \\
\quad \cdot\left\{-a d\left(\alpha\left(y_{1}\right), \cdots, \widehat{\alpha\left(y_{i}\right)}, \cdots, \alpha\left(y_{n}\right)\right) a d\left(x_{1}, \cdots, x_{n-2}, y_{i}\right)\right\}
\end{aligned}
$$

for all $i=1,2, \cdots, n$.
Proof. $(\Rightarrow)$ We firstly prove that the necessity holds. Then, by the definition of $\mathrm{ad}^{*}$, one gets

$$
\begin{aligned}
\mathrm{a} d^{*} & (\alpha(\mathscr{X})) \mathrm{a} d^{*}(\mathscr{Y})(f)(z) \\
& =-(-1)^{|\mathscr{X}|(|\mathscr{Y}|+|f|)} \mathrm{a} d^{*}(\mathscr{Y})(f)(\mathrm{a} d \alpha(\mathscr{X})(z)) \\
& =-(-1)^{|\mathscr{X}|(|\mathscr{Y}|+|f|)}\left(-(-1)^{|\mathscr{Y}||f|} f(\mathrm{a} d(\mathscr{Y}) \mathrm{a} d \alpha(\mathscr{X})(z))\right. \\
& =(-1)^{|\mathscr{X}|(|\mathscr{Y}|+|f|)+|\mathscr{Y}||f|} f(\mathrm{a} d(\mathscr{Y}) \mathrm{a} d \alpha(\mathscr{X})(z))
\end{aligned}
$$

and

$$
\begin{aligned}
(-1)^{|X||Y|} & \mathrm{a} d^{*} \alpha(\mathscr{Y}) \mathrm{a} d^{*}(\mathscr{X})(f)(z) \\
& =(-1)^{|\mathscr{X}||\mathscr{Y}|}\left(-(-1)^{|\mathscr{Y}|(|X|+|f|)} \mathrm{a} d^{*}(\mathscr{X})(f)(\mathrm{a} d \alpha(\mathscr{Y})(z))\right. \\
& =-(-1)^{|\mathscr{Y}||f|}\left(-(-1)^{|x||f|} f(\mathrm{a} d(\mathscr{X}) \mathrm{a} d \alpha(\mathscr{Y})(z))\right. \\
& =(-1)^{(|\mathscr{X}|+|\mathscr{Y}|)|f|} f(\mathrm{a} d(\mathscr{X}) \mathrm{a} d \alpha(\mathscr{Y})(z)) .
\end{aligned}
$$

Moreover, we have

$$
\begin{aligned}
\mathrm{a} d^{*} & \left([\mathscr{X}, \mathscr{Y}]_{\alpha}\right) \circ v(f)(z) \\
& =-(-1)^{(|\mathscr{X}|+|\mathscr{Y}|)|f|)} v(f)\left(\mathrm{a} d[\mathscr{X}, \mathscr{Y}]_{\alpha}(z)\right) \\
& =-(-1)^{(|\mathscr{X}|+|\mathscr{Y}|)|f|} f\left(\alpha \circ \mathrm{a} d[\mathscr{X}, \mathscr{Y}]_{\alpha}(z)\right) .
\end{aligned}
$$

By (13), we have ad(X)a $d \alpha(\mathscr{Y})-(-1)^{|\mathscr{X}||\mathscr{Y}|} \mathrm{a} d(\mathscr{Y}) \mathrm{a} d \alpha$ $(\mathscr{X})=\alpha \circ \mathrm{a} d[\mathscr{X}, \mathscr{Y}]_{\alpha}$.

$$
\begin{aligned}
\mathrm{a} d^{*}( & \left.\alpha\left(x_{1}\right), \cdots, \alpha\left(x_{n-2}\right),\left[y_{1}, \cdots, y_{n}\right]\right) v(f)(z) \\
= & -(-1)^{\left(\left|x_{1}\right|+\cdots+\left|x_{n-2}\right|+\left|y_{1}\right|+\cdots+\left|y_{n}\right|\right)|f|} f\left[\alpha\left(x_{1}\right), \cdots, \alpha\left(x_{n-2}\right),\right. \\
& \left.\cdot\left[y_{1}, \cdots, y_{n}\right], \alpha(z)\right] \\
= & -(-1)^{\left(\left|x_{1}\right|+\cdots+\left|x_{n-2}\right|+\left|y_{1}\right|+\cdots+\left|y_{n}\right|\right)|f|}\left(-(-1)^{|z|\left(\left|y_{1}\right|+\cdots+\left|y_{n}\right|\right)}\right) \\
& \cdot f\left[\alpha\left(x_{1}\right), \cdots, \alpha\left(x_{n-2}\right), \alpha(z),\left[y_{1}, \cdots, y_{n}\right]\right] \\
= & -(-1)^{\left(\left|x_{1}\right|+\cdots+\left|x_{n-2}\right|+\left|y_{1}\right|+\cdots+\left|y_{n}\right|\right)|f|}\left(-(-1)^{|z|\left(\left|y_{1}\right|+\cdots+\left|y_{n}\right|\right)}\right) \\
& \cdot \sum_{i=1}^{n}(-1)^{\left(\left|x_{1}\right|+\cdots+\left|x_{n-2}\right|+|z|\right)\left(\left|y_{1}\right|+\cdots+\left|y_{i-1}\right|\right)} \\
& \cdot f\left[\alpha\left(y_{1}\right), \cdots, \alpha\left(y_{i-1}\right),\left[x_{1}, \cdots, x_{n-2}, z, y_{i}\right], \alpha\left(y_{i+1}\right), \cdots, \alpha\left(y_{n}\right)\right] \\
=- & \sum_{i=1}^{n}(-1)^{n-i}(-1)^{\left|y_{i}\right|\left(\left|y_{i+1}\right|+\cdots+\left|y_{n}\right|\right)+\left(\left|x_{1}\right|+\cdots+\left|x_{n-2}\right|+\left|y_{1}\right|+\cdots+\mid y_{n}\right)|f|} \\
& \cdot(-1)^{\left(\left|x_{1}\right|+\cdots+\left|x_{n-2}\right|\right)\left(\left|y_{1}\right|+\cdots+\left|y_{i}\right| \wedge+\cdots+\left|y_{n}\right|\right)} f\left[\alpha\left(y_{1}\right), \cdots, \widehat{\alpha\left(y_{i}\right)}, \cdots, \alpha\left(y_{n}\right),\right. \\
& \left.\cdot\left[x_{1}, \cdots, x_{n-2}, y_{i}, z\right]\right] \\
=- & \sum_{i=1}^{n}(-1)^{n-i}(-1)^{\left|y_{i}\right|\left(\left|y_{i+1}\right|+\cdots+\left|y_{n}\right|\right)+\left(\left|x_{1}\right|+\cdots+\left|x_{n-2}\right|+\left|y_{1}\right|+\cdots+\left|y_{n}\right|\right)|f|} \\
& \cdot(-1)^{\left(\left|x_{1}\right|+\cdots+\left|x_{n-2}\right|\right)\left(\left|y_{1}\right|+\cdots+\left|y_{i}\right||\Lambda+\cdots+| y_{n} \mid\right)} \\
& \cdot f\left(\mathrm{a} d\left(\alpha\left(y_{1}\right), \cdots, \widehat{\alpha\left(y_{i}\right)}, \cdots, \alpha\left(y_{n}\right)\right) \mathrm{a} d\left(x_{1}, \cdots, x_{n-2}, y_{i}\right)(z)\right),
\end{aligned}
$$




$$
\begin{gathered}
\sum_{i=1}^{n}(-1)^{n-i}(-1)^{\left(\left|x_{1}\right|+\cdots+\left|x_{n-2}\right|\right)\left(\left|y_{1}\right|+\cdots+\left|y_{i}\right| \wedge+\cdots+\left|y_{n}\right|\right)+\left|y_{i}\right|\left(\left|y_{i+1}\right|+\cdots+\left|y_{n}\right|\right)} \\
\cdot \mathrm{a}^{*}\left(\alpha\left(y_{1}\right), \cdots, \widehat{\alpha\left(y_{i}\right)}, \cdots, \alpha\left(y_{n}\right)\right) \mathrm{a} d^{*}\left(x_{1}, \cdots, x_{n-2}, y_{i}\right)(f)(z) \\
=\sum_{i=1}^{n}(-1)^{n-i}(-1)^{\left(\left|x_{1}\right|+\cdots+\left|x_{n-2}\right|\right)\left(\left|y_{1}\right|+\cdots+\left|y_{i}\right| \wedge+\cdots+\left|y_{n}\right|\right)+\left|y_{i}\right|\left(\left|y_{i+1}\right|+\cdots+\left|y_{n}\right|\right)} \\
\cdot\left(-(-1)^{\left(\left|y_{1}\right|+\cdots+\left|y_{i}\right| \wedge+\cdots+\left|y_{n}\right|\right)\left(\left|x_{1}\right|+\cdots+\left|x_{n-2}\right|+\left|y_{i}\right|+|f|\right)}\right) \\
\cdot \cdot d^{*}\left(x_{1}, \cdots, x_{n-2}, y_{i}\right)(f)\left(\mathrm{a} d\left(\alpha\left(y_{1}\right), \cdots, \widehat{\alpha\left(y_{i}\right)}, \cdots, \alpha\left(y_{n}\right)\right)(z)\right) \\
\sum_{i=1}^{n}(-1)^{n-i}(-1)^{\left|y_{i}\right|\left(\left|y_{1}\right|+\cdots+\left|y_{i-1}\right|\right)+|f|\left(\left|y_{1}\right|+\cdots+\left|y_{n}\right|+\left|x_{1}\right|+\cdots+\left|x_{n-2}\right|\right)} \\
\cdot f\left(\mathrm{ad}\left(x_{1}, \cdots, x_{n-2}, y_{i}\right) \mathrm{ad}\left(\alpha\left(y_{1}\right), \cdots, \widehat{\alpha\left(y_{i}\right)}, \cdots, \alpha\left(y_{n}\right)\right)(z)\right) .
\end{gathered}
$$

By (14), we obtain

$$
\begin{aligned}
\mathrm{ad} & \left(x_{1}, \cdots, x_{n-2}, y_{i}\right) \mathrm{ad}\left(\alpha\left(y_{1}\right), \cdots, \widehat{\alpha\left(y_{i}\right)}, \cdots, \alpha\left(y_{n}\right)\right) \\
= & (-1)^{\left(\left|x_{1}\right|+\cdots+\left|x_{n-2}\right|\right)\left(\left|y_{1}\right|+\cdots+\left|y_{i}\right| \wedge+\cdots+\left|y_{n}\right|\right)} \\
& \cdot\left\{-\mathrm{ad}\left(\alpha\left(y_{1}\right), \cdots, \widehat{\alpha\left(y_{i}\right)}, \cdots, \alpha\left(y_{i}\right)\right) \mathrm{ad}\left(x_{1}, \cdots, x_{n-2}, y_{i}\right)\right\} .
\end{aligned}
$$

$(\Leftarrow)$ It is easy to see that the sufficiency holds. The proof is complete.

The representation $a d^{*}$ as defined in Lemma 17 is called the coadjoint representation of $\mathfrak{g}$. Let $\theta$ be a homogeneous $n$ -linear map from $\wedge^{n} \mathfrak{g}$ into $\mathfrak{g}^{*}$ of degree 0 . Now, we define a bracket on $\mathfrak{g} \oplus \mathfrak{g}^{*}$ :

$$
\begin{aligned}
& {\left[x_{1}+f_{1}, \cdots, x_{n}+f_{n}\right]_{\theta}=\left[x_{1}, \cdots, x_{n}\right]_{\mathfrak{g}}+\theta\left(x_{1}, \cdots, x_{n}\right)} \\
& \quad+\sum_{i=1}^{n}(-1)^{n-i}(-1)^{\left|x_{i}\right|\left(\left|x_{i+1}\right|+\cdots+\left|x_{n}\right|\right)} \mathrm{a} d^{*}\left(x_{1}, \cdots, \widehat{x}_{i}, \cdots, x_{n}\right) \cdot f_{i} .
\end{aligned}
$$

Theorem 18. Let $(\mathfrak{g},[\cdot, \cdots, \cdot], \alpha)$ be an $n$-ary multiplicative Hom-Nambu-Lie superalgebra. Assume that the coadjoint representation exists. Then, $\left(\mathfrak{g} \oplus \mathfrak{g}^{*},[\cdot, \cdots, \cdot]_{\theta}, \alpha^{\prime}\right)$ is an $n$-ary multiplicative Hom-Nambu-Lie superalgebra if and only if $\theta$ $\in Z^{1}\left(\mathfrak{g}, \mathfrak{g}^{*}\right)_{\overline{0}}$, where $\alpha^{\prime}(x+f)=\alpha(x)+f \circ \alpha, \forall x \in \mathfrak{g}, y \in \mathfrak{g}^{*}$.

Proof. It is clear that $[\cdot, \cdots, \cdot]_{\theta}$ satisfies (3) if and only if $\theta$ $\in C^{1}\left(\mathfrak{g}, \mathfrak{g}^{*}\right)_{\overline{0}}$. Let $\mathscr{X}+\mathscr{F}=\left(x_{1}+f_{1}\right) \wedge \cdots \wedge\left(x_{n-1}+f_{n-1}\right)$ and $\mathscr{Y}+\mathscr{G}=\left(y_{1}+g_{1}\right) \wedge \cdots \wedge\left(y_{n-1}+g_{n-1}\right)$. Then, we have

$$
\begin{aligned}
& \left(\alpha^{\prime}(\mathscr{X}+\mathscr{F})\right) \cdot\left((\mathscr{Y}+\mathscr{G}) \cdot\left(y_{n}+g_{n}\right)\right) \\
& =(\alpha(\mathscr{X})+\mathscr{F} \circ \alpha) \cdot\left\{\sum_{i=1}^{n}(-1)^{n-i}(-1)^{\left|y_{i}\right|\left(\left|y_{i+1}\right|+\cdots+\left|y_{n}\right|\right)} \mathrm{a} d^{*}\left(y_{1}, \cdots, \widehat{y}_{i}, \cdots, y_{n}\right) \cdot g_{i}+\mathscr{Y} \cdot y_{n}+\theta\left(\mathscr{Y}, y_{n}\right)\right\} \\
& =\alpha(\mathscr{X}) \cdot\left(\mathscr{Y} \cdot y_{n}\right)+\theta\left(\alpha(\mathscr{X}), \mathscr{Y} \cdot y_{n}\right)+\mathrm{a} d^{*}(\alpha(\mathscr{X})) \cdot \theta\left(\mathscr{Y}, y_{n}\right)+\sum_{j=1}^{n-1}(-1)^{n-j}(-1)^{\left|x_{j}\right|\left(\left|x_{j+1}\right|+\cdots+\left|x_{n-1}\right|+|\mathscr{y}|+\left|y_{n}\right|\right)} \mathrm{a} d^{*} \\
& \cdot\left(\alpha\left(x_{1}\right), \cdots, \widehat{\alpha\left(x_{j}\right)}, \cdots, \alpha\left(x_{n-1}\right), \mathscr{Y} \cdot y_{n}\right) \cdot\left(f_{j} \circ \alpha\right)+\sum_{i=1}^{n}(-1)^{n-i}(-1)^{\left|y_{i}\right|\left(\left|y_{i+1}\right|+\cdots+\left|y_{n}\right|\right)} \mathrm{a} d^{*}(\alpha(\mathscr{X})) \cdot\left(\mathrm{a} d^{*}\left(y_{1}, \cdots, \hat{y}_{i}, \cdots, y_{n}\right) \cdot g_{i}\right), \\
& \sum_{i=1}^{n}(-1)^{|\mathscr{X}|\left(\left|y_{1}\right|+\cdots+\left|y_{i-1}\right|\right)}\left[\alpha\left(y_{1}\right)+\left(g_{1} \circ \alpha\right), \cdots,(\mathscr{X}+\mathscr{F}) \cdot\left(y_{i}+g_{i}\right), \cdots, \alpha\left(y_{n}\right)+\left(g_{n} \circ \alpha\right)\right]_{\theta} \\
& =\sum_{i=1}^{n}(-1)^{|\mathscr{X}|\left(\left|y_{1}\right|+\cdots+\left|y_{i-1}\right|\right)}\left[\alpha\left(y_{1}\right)+\left(g_{1} \circ \alpha\right), \cdots,\left\{\mathscr{X} \cdot y_{i}+\theta\left(\mathscr{X}, y_{i}\right)+\mathrm{a} d^{*}(\mathscr{X}) \cdot g_{i}\right.\right. \\
& \left.\left.+\sum_{j=1}^{n-1}(-1)^{n-j}(-1)^{\left|x_{j}\right|\left(\left|x_{j+1}\right|+\cdots+\left|x_{n-1}\right|+\left|y_{i}\right|\right)} \mathrm{a} d^{*}\left(x_{1}, \cdots, \widehat{x}_{j}, \cdots, x_{n-1}, y_{i}\right) \cdot f_{j}\right\}, \cdots, \alpha\left(y_{n}\right)+\left(g_{n} \circ \alpha\right)\right]_{\theta} \\
& =\sum_{i=1}^{n}(-1)^{|\mathscr{X}|\left(\left|y_{1}\right|+\cdots+\left|y_{i-1}\right|\right)}\left\{\left[\alpha\left(y_{1}\right), \cdots, \mathscr{X} \cdot y_{i}, \cdots, \alpha\left(y_{n}\right)\right]+\theta\left(\alpha\left(y_{1}\right), \cdots, \mathscr{X} \cdot y_{i}, \cdots, \alpha\left(y_{n}\right)\right)\right. \\
& +\sum_{k<i}(-1)^{n-k}(-1)^{\left|y_{k}\right|\left(\left|y_{k+1}\right|+\cdots+\left|y_{n}\right|+|\mathscr{X}|\right)} \mathrm{a} d^{*}\left(\alpha\left(y_{1}\right), \cdots, \widehat{\alpha\left(y_{k}\right)}, \cdots, \mathscr{X} \cdot y_{i}, \cdots, \alpha\left(y_{n}\right)\right) \cdot\left(g_{k} \circ \alpha\right) \\
& +\sum_{i<k}(-1)^{n-k}(-1)^{\left|y_{k}\right|\left(\left|y_{k+1}\right|+\cdots+\left|y_{n}\right|\right)} \mathrm{a} d^{*}\left(\alpha\left(y_{1}\right), \cdots, \mathcal{X} \cdot y_{i}, \cdots, \widehat{\alpha\left(y_{k}\right)}, \cdots, \alpha\left(y_{n}\right)\right) \cdot\left(g_{k} \circ \alpha\right) \\
& +(-1)^{n-i}(-1)^{\left(|X|+\left|y_{i}\right|\right)\left(\left|y_{i+1}\right|+\cdots+\left|y_{n}\right|\right)} \mathrm{a} d^{*}\left(\alpha\left(y_{1}\right), \cdots, \widehat{\alpha\left(y_{i}\right)}, \cdots, \alpha\left(y_{n}\right)\right) \cdot\left\{\theta\left(\mathscr{X}, y_{i}\right)+\mathrm{ad}^{*}(\mathscr{X}) \cdot g_{i}\right. \\
& \left.\left.+\sum_{j=1}^{n-1}(-1)^{n-j}(-1)^{\left|x_{j}\right|\left(\left|x_{j+1}\right|+\cdots+\left|x_{n-1}\right|+\left|y_{i}\right|\right)} \mathrm{a} d^{*}\left(x_{1}, \cdots, \widehat{x}_{j}, \cdots, x_{n-1}, y_{i}\right) \cdot f_{j}\right\}\right\} .
\end{aligned}
$$


Since $[, \cdots,]_{\mathfrak{g}}$ satisfies $(4)$ and $d^{*}(\mathscr{X})$ satisfies $(14)$, it can be concluded that $[, \cdots,]_{\theta}$ satisfies (4) if and only if

$$
\begin{aligned}
0= & \theta\left(\alpha(\mathscr{X}), \mathscr{Y} \cdot y_{n}\right)+\mathrm{ad}^{*}(\alpha(\mathscr{X})) \cdot \theta\left(\mathscr{Y}, y_{n}\right) \\
& -\sum_{i=1}^{n}(-1)^{|\mathscr{X}|\left(\left|y_{1}\right|+\cdots+\left|y_{i-1}\right|\right)} \cdot \theta\left(\alpha\left(y_{1}\right), \cdots, \mathscr{X} \cdot y_{i}, \cdots, \alpha\left(y_{n}\right)\right) \\
& -\sum_{i=1}^{n}(-1)^{|\mathscr{X}|\left(\left|y_{1}\right|+\cdots+\left|y_{i-1}\right|\right)} \cdot(-1)^{n-i}(-1)^{\left(|\mathscr{X}|+\left|y_{i}\right|\right)\left(\left|y_{i+1}\right|+\cdots+\left|y_{n}\right|\right)} \\
& \cdot \mathrm{a} d^{*}\left(\alpha\left(y_{1}\right), \cdots, \widehat{\alpha\left(y_{i}\right)}, \cdots, \alpha\left(y_{n}\right)\right) \cdot \theta\left(\mathscr{X}, y_{i}\right) \\
= & \delta \theta\left(\mathscr{X}, \mathscr{Y}, y_{n}\right),
\end{aligned}
$$

i.e., $\theta \in Z^{1}\left(\mathfrak{g}, \mathfrak{g}^{*}\right)_{\overline{0}}$.

Definition 19. Let $(\mathfrak{g},[\cdot, \cdots, \cdot], \alpha)$ be an $n$-ary multiplicative Hom-Nambu-Lie superalgebra. A bilinear form $\langle,\rangle_{\mathfrak{g}}$ on $\mathfrak{g}$ is said to be nondegenerate if

$$
\mathfrak{g}^{\perp}=\left\{x \in \mathfrak{g} \mid\langle x, y\rangle_{\mathfrak{g}}=0, \forall y \in \mathfrak{g}\right\}=0 ;
$$

invariant if

$$
\begin{aligned}
\left\langle\left[x_{1}, \cdots, x_{n-1}, y\right]_{\mathfrak{g}}, z\right\rangle_{\mathfrak{g}}= & -(-1)^{\left(\left|x_{1}\right|+\cdots+\left|x_{n-1}\right|\right)|y|} \\
& \cdot\left\langle y,\left[x_{1}, \cdots, x_{n-1}, z\right]_{\mathfrak{g}}\right\rangle_{\mathfrak{g}} \\
& \forall x_{1}, \cdots, x_{n-1}, y, z \in \mathfrak{g} ;
\end{aligned}
$$

supersymmetric if

$$
\langle x, y\rangle_{\mathfrak{g}}=(-1)^{|x||y|}\langle y, x\rangle_{\mathfrak{g}}
$$

consistent if

$$
\langle x, y\rangle_{\mathfrak{g}}=0, \forall x, y \in \mathfrak{g},|x| \neq|y| ;
$$

$\alpha$ is called $\langle,\rangle_{\mathfrak{g}}$-symmetric, if

$$
\langle\alpha(x), y\rangle_{\mathfrak{g}}=\langle\alpha(y), x\rangle_{\mathfrak{g}}, \forall x, y \in \mathfrak{g} ;
$$

a subspace $I$ of $\mathfrak{g}$ is called isotropic if $I \subseteq I^{\perp}$.

In this section, we only consider consistent bilinear forms. If $\mathfrak{g}$ admits a nondegenerate invariant supersymmetric bilinear form $\langle,\rangle_{\mathfrak{g}}$ such that $\alpha$ is $\langle,\rangle_{\mathfrak{g}}$-symmetric, then we call $\left(\mathfrak{g},[\cdot, \cdots, \cdot]_{\mathfrak{g}}, \alpha,\langle,\rangle_{\mathfrak{g}}\right)$ a metric $n$-ary multiplicative HomNambu-Lie superalgebra. In particular, a metric vector space is a pair $(V, \alpha)$ consisting of a $\mathbb{Z}_{2}$-graded vector space $V=$ $V_{\overline{0}} \oplus V_{\overline{1}}$ and an endomorphism $\alpha$ of $V$ admitting a nondegenerate invariant supersymmetric bilinear form $\langle,\rangle_{\mathfrak{g}}$ such that $\alpha$ is $\langle,\rangle_{\mathfrak{g}}$-symmetric.
Lemma 20. With notations of Theorem 18, define a bilinear form $\langle,\rangle_{\theta}:\left(\mathfrak{g} \oplus \mathfrak{g}^{*}\right) \times\left(\mathfrak{g} \oplus \mathfrak{g}^{*}\right) \longrightarrow \mathbb{K}$ by

$$
\langle x+f, y+g\rangle_{\theta}=f(y)+(-1)^{|x||y|} g(x) .
$$

Then, $\langle y+g, x+f\rangle_{\theta}=(-1)^{|x||y|}\langle x+f, y+g\rangle_{\theta},\langle,\rangle_{\theta}$ is non-

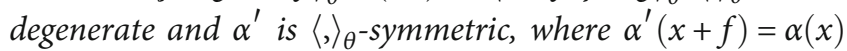
$+f \circ \alpha, x \in \mathfrak{g}, f \in \mathfrak{g}^{*}$. Moreover, $\left(\mathfrak{g} \oplus \mathfrak{g}^{*},[\cdot, \cdots, \cdot]_{\theta}, \alpha^{\prime},\langle,\rangle_{\theta}\right)$ is metric if and only if the following identity holds

$$
\theta(\mathscr{X}, y)(z)+(-1)^{|y||z|} \theta(\mathscr{X}, z)(y)=0 .
$$

Proof. If $x+f$ is orthogonal to all elements of $\mathfrak{g} \oplus \mathfrak{g}^{*}$, then for arbitrary element $y+g \in \mathfrak{g} \oplus \mathfrak{g}^{*}$, we have $f(y)=0$ and $(-1)^{|x||y|} g(x)=0$, which implies that $x=0$ and $f=0$, so $\langle,\rangle_{\theta}$ is nondegenerate. Moreover, we have

$$
\begin{aligned}
\langle y+g, x+f\rangle_{\theta} & =g(x)+(-1)^{|y||x|} f(y) \\
& =(-1)^{|x||y|}\left(f(y)+(-1)^{|x||y|} g(x)\right) \\
& =(-1)^{|x||y|}\langle x+f, y+g\rangle_{\theta} .
\end{aligned}
$$

In addition, one gets

$$
\begin{aligned}
\left\langle\alpha^{\prime}(x+f), y+g\right\rangle_{\theta} & =\langle\alpha(x)+f \circ \alpha, y+g\rangle_{\theta} \\
& =f \circ \alpha(y)+(-1)^{|x| y \mid} g(\alpha(x)), \\
\left\langle x+f, \alpha^{\prime}(y+g)\right\rangle_{\theta} & =\langle x+f, \alpha(y)+g \circ \alpha\rangle_{\theta} \\
& =f \alpha(y)+(-1)^{|x||y|} g \circ \alpha(x) .
\end{aligned}
$$

Hence, $\left\langle\alpha^{\prime}(x+f), y+g\right\rangle_{\theta}=\left\langle x+f, \alpha^{\prime}(y+g)\right\rangle_{\theta}$.

Furthermore, $\left(\mathfrak{g} \oplus \mathfrak{g}^{*},\langle,\rangle_{\theta}\right)$ is metric if and only if

$$
\begin{aligned}
0= & \langle(\mathscr{X}+\mathscr{F}) \cdot(y+g), z+h\rangle_{\theta} \\
& +(-1)^{|\mathscr{X}||y|}\langle y+g,(\mathscr{X}+\mathscr{F}) \cdot(z+h)\rangle_{\theta} \\
= & \left\langle\mathscr{X} \cdot y+\theta(\mathscr{X}, y)+\mathrm{a} d^{*}(\mathscr{X}) \cdot g, z+h\right\rangle_{\theta} \\
& +\left\langle\sum_{i=1}^{n-1}(-1)^{n-i}(-1)^{\left|x_{i}\right|\left(\left|x_{i+1}\right|+\cdots+\left|x_{n-1}\right|+|y|\right)} \mathrm{a} d^{*}\right. \\
& \left.\cdot\left(x_{1}, \cdots, \widehat{x}_{i}, \cdots, x_{n-1}, y\right) \cdot f_{i}, z+h\right\rangle_{\theta} \\
& +(-1)^{|\mathscr{X}||y|}\left\langle y+g, \mathscr{X} \cdot z+\theta(\mathscr{X}, z)+\mathrm{a} d^{*}(\mathscr{X}) \cdot h\right\rangle_{\theta} \\
& +(-1)^{|X||y|}\left\langle y+g, \sum_{i=1}^{n-1}(-1)^{n-i}(-1)^{\left|x_{i}\right|\left(\left|x_{i+1}\right|+\cdots+\left|x_{n-1}\right|+|z|\right)} \mathrm{a} d^{*}\right. \\
& \left.\cdot\left(x_{1}, \cdots, \widehat{x}_{i}, \cdots, x_{n-1}, z\right) \cdot f_{i}\right\rangle \\
= & \theta(\mathscr{X}, y)(z)+(-1)^{|y||z|} \theta(\mathscr{X}, z)(y),
\end{aligned}
$$

i.e., (45) holds. 
Now, we give the definition of $T^{*}$-extensions.

Definition 21. For a 1-supercocycle $\theta$ satisfying (45) we shall call the metric $n$-ary multiplicative Hom-Nambu-Lie superalgebra $\left(\mathfrak{g} \oplus \mathfrak{g}^{*},[\cdot, \cdots, \cdot]_{\theta}, \alpha^{\prime},\langle,\rangle_{\theta}\right)$ the $T^{*}$-extension of $(\mathfrak{g},[\cdot, \cdots, \cdot], \alpha)($ by $\theta)$ and denote it by $\left(T_{\theta}^{*} \mathfrak{g},[\cdot, \cdots, \cdot]_{\theta}, \alpha^{\prime}\right)$.

Theorem 22. Let $(\mathfrak{g},[\cdot, \cdots, \cdot], \alpha)$ be an $n$-ary multiplicative Hom-Nambu-Lie superalgebra over a field $\mathbb{K}$. Let

$$
\begin{aligned}
\mathfrak{g}^{(0)} & =\mathfrak{g}, \mathfrak{g}^{(m+1)}=\left[\mathfrak{g}^{(m)}, \cdots, \mathfrak{g}^{(m)}\right]_{\mathfrak{g}} \text { and } \mathfrak{g}^{1}=\mathfrak{g}, \mathfrak{g}^{m+1} \\
& =\left[\mathfrak{g}^{m}, \mathfrak{g}, \cdots, \mathfrak{g}\right]_{\mathfrak{g}}, \forall m \geq 0 .
\end{aligned}
$$

$\mathfrak{g}$ is called solvable (nilpotent) of length $k$ if and only if there is a smallest integer $k$ such that $\mathfrak{g}^{(k)}=0\left(\mathfrak{g}^{k}=0\right)$. Then

(1) If $\mathfrak{g}$ is solvable of length $k$, then $T_{\theta}^{*} \mathfrak{g}$ is solvable of length $k$ or $k+1$.

(2) If $\mathfrak{g}$ is nilpotent of length $k$, then $T_{\theta}^{*} \mathfrak{g}$ is nilpotent of length at least $k$ and at most $2 k-1$. In particular, the nilpotent length of $T_{0}^{*} \mathfrak{g}$ is $k$.

(3) If $\mathfrak{g}$ can be decomposed into a direct sum of two Homideals of $\mathfrak{g}$, then $T_{0}^{*} \mathfrak{g}$ can be too.

Proof. (1) Suppose that $\mathfrak{g}$ is solvable of length $k$. Since $\left(T_{\theta}^{*} \mathfrak{g}\right)^{(m)} / \mathfrak{g}^{*} \cong \mathfrak{g}^{(m)}$ and $\mathfrak{g}^{(k)}=0$, we have $\left(T_{\theta}^{*} \mathfrak{g}\right)^{(k)} \subseteq \mathfrak{g}^{*}$, which implies $\left(T_{\theta}^{*} \mathfrak{g}\right)^{(k+1)}=0$ because $\mathfrak{g}^{*}$ is abelian, and it follows that $T_{\theta}^{*} \mathfrak{g}$ is solvable of length $k$ or $k+1$.

(2) Suppose that $\mathfrak{g}$ is nilpotent of length $k$. Since $\left(T_{\theta}^{*} \mathfrak{g}\right)^{m}$ $/ \mathfrak{g}^{*} \cong \mathfrak{g}^{m}$ and $\mathfrak{g}^{k}=0$, we have $\left(T_{\theta}^{*} \mathfrak{g}\right)^{k} \subseteq \mathfrak{g}^{*}$. Let $f \in\left(T_{\theta}^{*} \mathfrak{g}\right)^{k} \subseteq$ $\mathfrak{g}^{*}, y \in \mathfrak{g}, \quad \mathscr{X}_{j}+\mathscr{F}_{j}=\left(\mathscr{X}_{j}^{1}+\mathscr{F}_{j}^{1}\right) \wedge \cdots \wedge\left(\mathscr{X}_{j}^{n-1}+\mathscr{F}_{j}^{n-1}\right) \in$ $\left(T_{\theta}^{*} \mathfrak{g}\right)^{\wedge^{n-1}}, j=1, \cdots, k-1$. Then

$$
\begin{aligned}
& \left(\left(\mathscr{X}_{1}+\mathscr{F}_{1}\right) \cdots\left(\mathscr{X}_{k-1}+\mathscr{F}_{k-1}\right) \cdot f\right)(y) \\
& \quad=\left(\mathrm{a}^{*}\left(\mathscr{X}_{1}\right) \cdots \mathrm{a} d^{*}\left(\mathscr{X}_{k-1}\right) \cdot f\right)(y) \in f\left(\mathfrak{g}^{k}\right)=0 .
\end{aligned}
$$

This proves that $\left(T_{\theta}^{*} \mathfrak{g}\right)^{2 k-1}=0$. Hence $T_{\theta}^{*} \mathfrak{g}$ is nilpotent of length at least $k$ and at most $2 k-1$.

Now consider the case of trivial $T^{*}$-extension $T_{0}^{*} \mathfrak{g}$ of $\mathfrak{g}$. Note that

$$
\begin{aligned}
& \left(\mathscr{X}_{1}+\mathscr{F}_{1}\right) \cdots\left(\mathscr{X}_{k-1}+\mathscr{F}_{k-1}\right) \cdot(y+g) \\
& =\mathrm{a} d\left(X_{1}\right) \cdots \mathrm{a} d\left(X_{k-1}\right) \cdot y+\mathrm{ad}^{*}\left(X_{1}\right) \cdots \mathrm{a} d^{*}\left(X_{k-1}\right) \cdot g
\end{aligned}
$$



$$
\begin{aligned}
& \cdot\left(X_{1}\right) \cdots \mathrm{ad}^{*}\left(X_{j-1}\right) \mathrm{ad} d^{*}\left(X_{j}^{1}, \cdots, \widehat{X_{j}^{i}}, \cdots, X_{j}^{n-1}, \mathrm{ad}\left(X_{j+1}\right) \cdots \mathrm{a} d\right. \\
& \left.\cdot\left(\mathscr{X}_{k-1}\right) \cdot y\right) \cdot \mathscr{F}_{j}^{i}=0 \text {. }
\end{aligned}
$$

Then, $\left(T_{\theta}^{*} \mathfrak{g}\right)^{k}=0$, as required.
(3) Suppose that $0 \neq \mathfrak{g}=I \oplus J$, where $I$ and $J$ are two nonzero Hom-ideals of $\mathfrak{g}$. Let $I^{*}=\left\{f \in \mathfrak{g}^{*} \mid f(J)=0\right\}$ and $J^{*}=\left\{f \in \mathfrak{g}^{*} \mid f(I)=0\right\}$. Then, $I^{*}$ (resp. $\left.J^{*}\right)$ can canonically be identified with the dual space of $I$ (resp. $J$ ) and $\mathfrak{g}^{*} \cong I^{*}$ $\oplus J^{*}$.

Note that

$$
\begin{aligned}
{\left[T_{0}^{*} I, T_{0}^{*} \mathfrak{g}, \cdots, T_{0}^{*} \mathfrak{g}\right]_{0}=} & {\left[I \oplus I^{*}, \mathfrak{g} \oplus \mathfrak{g}^{*}, \cdots, \mathfrak{g} \oplus \mathfrak{g}^{*}\right]_{0} } \\
= & {[I, \mathfrak{g}, \cdots, \mathfrak{g}]_{\mathfrak{g}}+\left[I^{*}, \mathfrak{g}, \cdots, \mathfrak{g}\right]_{0} } \\
& +\left[I, \mathfrak{g}, \cdots, \mathfrak{g}, \mathfrak{g}^{*}\right]_{0} \subseteq I \oplus I^{*}=T_{0}^{*} I,
\end{aligned}
$$

since

$$
\begin{aligned}
{\left[I^{*}, \mathfrak{g}, \cdots, \mathfrak{g}\right]_{0}(J) } & =I^{*}\left([J, \mathfrak{g}, \cdots, \mathfrak{g}]_{\mathfrak{g}}\right) \subseteq I^{*}(J) \\
& =0 \text { and }\left[I, \mathfrak{g}, \cdots, \mathfrak{g}, \mathfrak{g}^{*}\right]_{0}(J) \\
& =\mathfrak{g}^{*}\left([I, J, \mathfrak{g}, \cdots, \mathfrak{g}]_{\mathfrak{g}}\right)=\mathfrak{g}^{*}(0)=0 .
\end{aligned}
$$

Moreover, for $x+f \in T_{0}^{*} I=I \oplus I^{*}$, we have $\alpha^{\prime}(x+f)=\alpha$ $(x)+f \circ \alpha \in I \oplus I^{*}$ since $f \circ \alpha \in \mathfrak{g}^{*}$ and $f \circ \alpha(J) \in f(J)=0$, that is, $\alpha^{\prime}\left(T_{0}^{*} I\right) \subseteq T_{0}^{*} I$. Then, $T_{0}^{*} I$ is a Hom-ideal of $T_{0}^{*} \mathfrak{g}$ and so is $T_{0}^{*} J$ in the same way. Hence, $T_{0}^{*} \mathfrak{g}$ can be decomposed into the direct sum $T_{0}^{*} I \oplus T_{0}^{*} J$ of two nonzero Homideals of $T_{0}^{*} \mathfrak{g}$.

Lemma 23. Let $\left(\mathfrak{g},[\cdot, \cdots, \cdot], \alpha,\langle,\rangle_{\theta}\right)$ be a metric n-ary multiplicative Hom-Nambu-Lie superalgebra of even dimension $m$ over a field $\mathbb{K}$ and $I$ be an isotropic $m / 2$-dimensional Homideal of $\mathfrak{g}$. Then, $I$ is abelian.

Proof. Since $\operatorname{dim} I+\operatorname{dim} I^{\perp}=m / 2+\operatorname{dim} I^{\perp}=m$ and $I \subseteq I^{\perp}$, we have $I=I^{\perp}$.

By $I$ is a Hom-ideal of $\mathfrak{g}$, one gets

$$
\left\langle\mathfrak{g},[\mathfrak{g}, \cdots, \mathfrak{g}, I, I]_{\mathfrak{g}}\right\rangle_{\theta}=\left\langle[\mathfrak{g}, \cdots, \mathfrak{g}, I]_{\mathfrak{g}}, I\right\rangle_{\theta} \subseteq\langle I, I\rangle_{\theta}=0,
$$

which implies $[\mathfrak{g}, \cdots, \mathfrak{g}, I, I]_{\mathfrak{g}} \subseteq \mathfrak{g}^{\perp}=0$.

Definition 24. Let $\left(\mathfrak{g},[\cdot, \cdots, \cdot]_{\mathfrak{g}}, \alpha\right)$ and $\left(\mathfrak{g}^{\prime},[\cdot, \cdots, \cdot]_{\mathfrak{g}}^{\prime}, \beta\right)$ be two $n$-ary Hom-Nambu-Lie superalgebras. A bijective homomorphism $\phi: \mathfrak{g} \longrightarrow \mathfrak{g}^{\prime}$ is called an isomorphism of $n$-ary Hom-Nambu-Lie superalgebras.

Definition 25. Two metric $n$-ary multiplicative Hom-NambuLie superalgebras $\left(\mathfrak{g},[\cdot, \cdots, \cdot]_{\mathfrak{g}}, \alpha,\langle,\rangle_{\mathfrak{g}}\right)$ and $\left(\mathfrak{g}^{\prime},[\cdot, \cdots, \cdot]_{\mathfrak{g}}{ }^{\prime}, \beta\right.$, $\left.\langle,\rangle_{\mathfrak{g}}{ }^{\prime}\right)$ is said to be isometric if there exists an $n$-ary multiplicative Hom-Nambu-Lie superalgebra isomorphism $\phi: \mathfrak{g}$ $\longrightarrow \mathfrak{g}^{\prime}$ such that $\langle x, y\rangle_{\mathfrak{g}}=\langle\phi(x), \phi(y)\rangle_{\mathfrak{g}}{ }^{\prime}, \forall x, y \in \mathfrak{g}$.

Theorem 26. Let $\left(\mathfrak{g},[\cdot, \cdots, \cdot]_{\mathfrak{g}}, \beta,\langle,\rangle_{\mathfrak{g}}\right)$ be a metric $n$-ary multiplicative Hom-Nambu-Lie superalgebra of dimension $m$ over a field $\mathbb{K}$ of characteristic not 2. Suppose that $\left(T_{\theta}^{*} \mathfrak{g}_{1}\right.$, $\left.[\cdot, \cdots, \cdot]_{\theta}, \alpha^{\prime},\langle,\rangle_{\theta}\right)$ is a $T^{*}$-extension of $\left(\mathfrak{g}_{1},[\cdot, \cdots, \cdot]_{\mathfrak{g}_{1}}, \alpha\right)$. 
Then, $\left(\mathfrak{g},[\cdot, \cdots, \cdot]_{\mathfrak{g}}, \beta,\langle,\rangle_{\mathfrak{g}}\right)$ is isometric to $\left(T_{\theta}^{*} \mathfrak{g}_{1},[\cdot, \cdots, \cdot]_{\theta}\right.$, $\left.\alpha^{\prime},\langle,\rangle_{\theta}\right)$ if and only if $m$ is even and $\mathfrak{g}$ contains an isotropic Hom-ideal I of dimension $m / 2$. In particular, $\mathfrak{g}_{1} \cong \mathfrak{g} / I$.

Proof. $(\Rightarrow)$ Since $\operatorname{dim} \mathfrak{g}_{1}=\operatorname{dim} \mathfrak{g}_{1}^{*}, \operatorname{dim} \mathfrak{g}=\operatorname{dim} T_{\theta}^{*} \mathfrak{g}_{1}=m$ is even. Moreover, $\alpha^{\prime}(f)=f \circ \alpha \in \mathfrak{g}_{1}^{*}$ for all $f \in \mathfrak{g}_{1}^{*}$. It is clear that $\mathfrak{g}_{1}^{*}$ is a Hom-ideal of dimension $m / 2$ and by the definition of $\langle,\rangle_{\theta}$, we have $\left\langle\mathfrak{g}_{1}^{*}, \mathfrak{g}_{1}^{*}\right\rangle_{\theta}=0$, i.e., $\mathfrak{g}_{1}^{*}$ is isotropic.

$(\Leftarrow)$ Suppose that $I$ is an $m / 2$-dimensional isotropic graded ideal of $\mathfrak{g}$. By Lemma 23, $I$ is abelian. Let $\mathfrak{g}_{1}=\mathfrak{g}$ / $I$ and $\pi: \mathfrak{g} \longrightarrow \mathfrak{g}_{1}$ be the canonical projection. Since ch $\mathbb{K}$ $\neq 2$, we can choose a complement graded subspace $\mathfrak{g}_{0} \subseteq$ $\mathfrak{g}$ such that $\mathfrak{g}=\mathfrak{g}_{0}+I$ and $\mathfrak{g}_{0} \subseteq \mathfrak{g}_{0}^{\perp}$. Then, $\mathfrak{g}_{0}^{\perp}=\mathfrak{g}_{0}$ since $\operatorname{dim} \mathfrak{g}_{0}=m / 2$.

Denote by $p_{0}$ (resp. $p_{1}$ ) the projection $\mathfrak{g} \longrightarrow \mathfrak{g}_{0}$ (resp. $\mathfrak{g} \longrightarrow I$ ) and let $f_{1}^{*}$ denote the homogeneous linear map $I \longrightarrow \mathfrak{g}_{1}^{*}: z \mapsto f_{1}^{*}(z)$, where $f_{1}^{*}(z)(\pi(x)):=\langle z, x\rangle_{\mathfrak{g}}, \forall x \in \mathfrak{g}, \forall$ $z \in I$.

If $\pi(x)=\pi(y)$, then $x-y \in I$, hence $\langle z, x-y\rangle_{\mathfrak{g}} \in\langle z, I\rangle_{\mathfrak{g}}$ $=0$ and so $\langle z, x\rangle_{\mathfrak{g}}=\langle z, y\rangle_{\mathfrak{g}}$, which implies $f_{1}^{*}$ is well defined. Moreover, $f_{1}^{*}$ is bijective and $\left|f_{1}^{*}(z)\right|=|z|$ for all $z \in I$.

In addition, $f_{1}^{*}$ has the following property:

$$
\begin{gathered}
f_{1}^{*}\left(\left[x_{1}, \cdots, z_{k}, \cdots, x_{n}\right]_{\mathfrak{g}}\right)(\pi(y))=(-1)^{n-k}(-1)^{\left|z_{k}\right|\left(\left|x_{k+1}\right|+\cdots+\left|x_{n}\right|\right)} \mathrm{a} d^{*} \\
\cdot\left(\pi\left(x_{1}\right), \cdots, \widehat{\pi\left(x_{k}\right)}, \cdots, \pi\left(x_{n}\right)\right) \cdot f_{1}^{*}\left(z_{k}\right)(\pi(y)),
\end{gathered}
$$

where $x_{1}, \cdots, x_{k-1}, x_{k+1}, \cdots, x_{n} \in \mathfrak{g}, z_{k} \in I$.

Define a homogeneous $n$-linear map

$$
\begin{aligned}
\theta: \mathfrak{g}_{1} \times \cdots \times \mathfrak{g}_{1} & \longrightarrow \mathfrak{g}_{1}^{*} \\
\left(\pi\left(x_{1}\right), \cdots, \pi\left(x_{n}\right)\right) & \mapsto f_{1}^{*}\left(p_{1}\left(\left[x_{1}, \cdots, x_{n}\right]_{\mathfrak{g}}\right)\right),
\end{aligned}
$$

where $x_{1}, \cdots, x_{n} \in \mathfrak{g}_{0}$. Then, $\theta$ is well defined since $\left.\pi\right|_{\mathfrak{g}_{0}}: \mathfrak{g}_{0}$ $\longrightarrow \mathfrak{g}_{0} / I \cong \mathfrak{g} / I=\mathfrak{g}_{1}$ is a linear isomorphism and $\theta \in C^{1}$ $\left(\mathfrak{g}_{1}, \mathfrak{g}_{1}^{*}\right)_{\overline{0}}$.

Now, define the bracket on $\mathfrak{g}_{1} \oplus \mathfrak{g}_{1}^{*}$ by (35), then, $\left(\mathfrak{g}_{1} \oplus \mathfrak{g}_{1}^{*}\right.$ ,$\left.\alpha^{\prime}\right)$ is a metric $n$-ary multiplicative Hom-Nambu-Lie superalgebra. Let $\varphi$ be a linear map $\mathfrak{g} \longrightarrow \mathfrak{g}_{1} \oplus \mathfrak{g}_{1}^{*}$ defined by $\varphi(x+z)$ $=\pi(x)+f_{1}^{*}(z), \forall x+z \in \mathfrak{g}=\mathfrak{g}_{0}+I$. Since $\left.\pi\right|_{\mathfrak{g}_{0}}$ and $f_{1}^{*}$ are linear isomorphisms, $\varphi$ is also a linear isomorphism. Note that

$$
\begin{aligned}
\varphi( & {\left.\left[x_{1}+z_{1}, \cdots, x_{n}+z_{n}\right]_{\mathfrak{g}}\right) } \\
= & \varphi\left(\left[x_{1}, \cdots, x_{n}\right]_{\mathfrak{g}}+\sum_{k=1}^{n}\left[x_{1}, \cdots, z_{k}, \cdots, x_{n}\right]_{\mathfrak{g}}\right) \\
= & \varphi\left(p_{0}\left(\left[x_{1}, \cdots, x_{n}\right]_{\mathfrak{g}}\right)+p_{1}\left(\left[x_{1}, \cdots, x_{n}\right]_{\mathfrak{g}}\right)\right. \\
& \left.+\sum_{k=1}^{n}\left[x_{1}, \cdots, z_{k}, \cdots, x_{n}\right]_{\mathfrak{g}}\right)=\pi\left(\left[x_{1}, \cdots, x_{n}\right]_{\mathfrak{g}}\right)
\end{aligned}
$$

$$
\begin{aligned}
& +f_{1}^{*}\left(p_{1}\left(\left[x_{1}, \cdots, x_{n}\right]_{\mathfrak{g}}\right)+\sum_{k=1}^{n}\left[x_{1}, \cdots, z_{k}, \cdots, x_{n}\right]_{\mathfrak{g}}\right) \\
= & {\left[\pi\left(x_{1}\right), \cdots, \pi\left(x_{n}\right)\right]_{\mathfrak{g}_{1}}+\theta\left(\pi\left(x_{1}\right), \cdots, \pi\left(x_{n}\right)\right) } \\
& +\sum_{k=1}^{n}(-1)^{n-k}(-1)^{\left|z_{k}\right|\left(\left|x_{k+1}\right|+\cdots+\left|x_{n}\right|\right)} \mathrm{a} d^{*} \\
& \cdot\left(\pi\left(x_{1}\right), \cdots, \widehat{\pi\left(x_{k}\right)}, \cdots, \pi\left(x_{n}\right)\right) \cdot f_{1}^{*}\left(z_{k}\right) \\
= & {\left[\pi\left(x_{1}\right)+f_{1}^{*}\left(z_{1}\right), \cdots, \pi\left(x_{n}\right)+f_{1}^{*}\left(z_{n}\right)\right]_{\theta} } \\
= & {\left[\varphi\left(x_{1}+z_{1}\right), \cdots, \varphi\left(x_{n}+z_{n}\right)\right]_{\theta}, }
\end{aligned}
$$

where we use the definitions of $\varphi$ and $\theta$ and (56). Moreover, $\varphi$ $\circ \alpha=\alpha^{\prime} \circ \varphi$. In fact, for $x+z \in \mathfrak{g}=\mathfrak{g}_{0}+I$, then,

$$
\begin{aligned}
\varphi \circ \alpha(x+z) & =\varphi(\alpha(x)+\alpha(z))=\pi(\alpha(x))+f_{1}^{*}(\alpha(z)) \\
\alpha^{\prime} \circ \varphi(x+z) & =\alpha^{\prime}\left(\pi(x)+f_{1}^{*}(z)\right)=\alpha(\pi(x))+f_{1}^{*}(z) \circ \alpha .
\end{aligned}
$$

Moreover,

$$
\begin{aligned}
f_{1}^{*}(z) \circ \alpha(\pi(x)) & =f_{1}^{*}(z) \pi(\alpha(x))=\langle z, \alpha(x)\rangle_{\mathfrak{g}} \\
& =\langle\alpha(z), x\rangle_{\mathfrak{g}}=f_{1}^{*}(\alpha(z))(\pi(x)) .
\end{aligned}
$$

Therefore, $f_{1}^{*}(z) \circ \alpha=f_{1}^{*}(\alpha(z))$, one gets $\varphi \circ \alpha=\alpha^{\prime} \circ \varphi$. Then, $\varphi$ is an isomorphism of $n$-ary multiplicative HomNambu-Lie superalgebras, hence, $\mathfrak{g}_{1} \oplus \mathfrak{g}_{1}^{*}$ is an $n$-ary multiplicative Hom-Nambu-Lie superalgebra. Furthermore, we have

$$
\begin{aligned}
& \left\langle\varphi\left(x_{0}+z\right), \varphi\left(x_{0}{ }^{\prime}+z^{\prime}\right)\right\rangle_{\theta} \\
& \quad=\left\langle\pi\left(x_{0}\right)+f_{1}^{*}(z), \pi\left(x_{0}{ }^{\prime}\right)+f_{1}^{*}\left(z^{\prime}\right)\right\rangle_{\theta} \\
& \quad=f_{1}^{*}(z)\left(\pi\left(x_{0}{ }^{\prime}\right)\right)+(-1)^{\left|x_{0}\right|\left|x_{0^{\prime}}\right|} f_{1}^{*}\left(z^{\prime}\right)\left(\pi\left(x_{0}\right)\right) \\
& \quad=\left\langle z, x_{0}{ }^{\prime}\right\rangle_{\mathfrak{g}}+(-1)^{\left|x_{0}\right|\left|x_{0^{\prime}}\right|}\left\langle z^{\prime}, x_{0}\right\rangle_{\mathfrak{g}}=\left\langle x_{0}+z, x_{0}{ }^{\prime}+z^{\prime}\right\rangle_{\mathfrak{g}},
\end{aligned}
$$

then, $\varphi$ is isometric. The relation

$$
\begin{aligned}
\langle[ & \left.\left.\varphi\left(x_{1}+z_{1}\right), \cdots, \varphi\left(x_{n}+z_{n}\right)\right]_{\theta}, \varphi\left(x_{n+1}+z_{n+1}\right)\right\rangle_{\theta} \\
= & \left\langle\varphi\left(\left[x_{1}+z_{1}, \cdots, x_{n}+z_{n}\right]_{\mathfrak{g}}\right), \varphi\left(x_{n+1}+z_{n+1}\right)\right\rangle_{\theta} \\
= & \left\langle\left[x_{1}+z_{1}, \cdots, x_{n}+z_{n}\right]_{\mathfrak{g}}, x_{n+1}+z_{n+1}\right\rangle_{\mathfrak{g}} \\
= & -(-1)^{\left(\left|x_{1}\right|+\cdots+\left|x_{n-1}\right|\right)\left|x_{n}\right|}\left\langle x_{n}+z_{n},\left[x_{1}+z_{1}, \cdots, x_{n-1}\right.\right. \\
& \left.\left.+z_{n-1}, x_{n+1}+z_{n+1}\right]_{\mathfrak{g}}\right\rangle_{\mathfrak{g}} \\
= & -(-1)^{\left(\left|x_{1}\right|+\cdots+\left|x_{n-1}\right|\right)\left|x_{n}\right|}\left\langle\varphi\left(x_{n}+z_{n}\right),\left[\varphi\left(x_{1}+z_{1}\right), \cdots,\right.\right. \\
& \left.\left.\cdot \varphi\left(x_{n-1}+z_{n-1}\right), \varphi\left(x_{n+1}+z_{n+1}\right)\right]_{\theta}\right\rangle_{\theta},
\end{aligned}
$$

implies that $\langle,\rangle_{\theta}$ on $\mathfrak{g}_{1} \oplus \mathfrak{g}_{1}^{*}$ is invariant. 
For $x+f, y+g \in \mathfrak{g}_{1} \oplus \mathfrak{g}_{1}^{*}$, then, there exist $x^{\prime}+z_{1}, y^{\prime}+$ $z_{2} \in \mathfrak{g}_{0}+I$ such that $\varphi\left(x^{\prime}+z_{1}\right)=x+f$ and $\varphi\left(y^{\prime}+z_{2}\right)=y+$ g. Hence, we have

$$
\begin{aligned}
\left\langle\alpha^{\prime}\right. & (x+f), y+g\rangle_{\theta} \\
& =\left\langle\alpha^{\prime}\left(\varphi\left(x^{\prime}+z_{1}\right)\right), \varphi\left(y^{\prime}+z_{2}\right)\right\rangle_{\theta} \\
& =\left\langle\varphi\left(\alpha\left(x^{\prime}+z_{1}\right)\right), \varphi\left(y^{\prime}+z_{2}\right)\right\rangle_{\theta} \\
& =\left\langle\alpha\left(x^{\prime}+z_{1}\right), y^{\prime}+z_{2}\right\rangle_{\theta} \\
& =\left\langle\alpha\left(y^{\prime}+z_{2}\right), x^{\prime}+z_{1}\right\rangle_{\theta} \\
& =\left\langle\varphi\left(\alpha\left(y^{\prime}+z_{2}\right)\right), \varphi\left(x^{\prime}+z_{1}\right)\right\rangle_{\theta} \\
& =\left\langle\alpha^{\prime}\left(\varphi\left(y^{\prime}+z_{2}\right)\right), \varphi\left(x^{\prime}+z_{1}\right)\right\rangle_{\theta} \\
& =\left\langle\alpha^{\prime}(y+g), x+f\right\rangle_{\theta}
\end{aligned}
$$

Therefore, $\left(\mathfrak{g}_{1} \oplus \mathfrak{g}_{1}^{*},[\cdot, \cdots, \cdot]_{\theta}, \alpha^{\prime},\langle,\rangle_{\theta}\right)$ is a metric $n$-ary multiplicative Hom-Nambu-Lie superalgebra. In this way, we get a $T^{*}$-extension $\left(T_{\theta}^{*} \mathfrak{g}_{1},[\cdot, \cdots, \cdot]_{\theta}, \alpha^{\prime},\langle,\rangle_{\theta}\right)$ of $\left(\mathfrak{g}_{1}\right.$, $\left.[\cdot, \cdots, \cdot]_{\mathfrak{g}_{1}}, \alpha\right)$ and consequently, $\left(\mathfrak{g},[\cdot, \cdots, \cdot]_{\mathfrak{g}}, \beta,\langle,\rangle_{\mathfrak{g}}\right)$ and $\left(T_{\theta}^{*}\right.$ $\left.\mathfrak{g}_{1},[\cdot, \cdots, \cdot]_{\theta}, \alpha^{\prime},\langle,\rangle_{\theta}\right)$ are isometric as required.

Suppose that $(\mathfrak{g},[\cdot, \cdots, \cdot], \alpha)$ is an $n$-ary multiplicative Hom-Nambu-Lie superalgebra and $\theta_{1}, \theta_{2} \in Z^{1}\left(\mathfrak{g}, \mathfrak{g}^{*}\right)_{\overline{0}}$ satisfying (45). $T_{\theta_{1}}^{*} \mathfrak{g}$ and $T_{\theta_{2}}^{*} \mathfrak{g}$ are said to be equivalent if there exists an isomorphism of $n$-ary multiplicative Hom-Nambu-Lie superalgebras $\phi: T_{\theta_{1}}^{*} \mathfrak{g} \longrightarrow T_{\theta_{2}}^{*} \mathfrak{g}$ such that $\left.\phi\right|_{\mathfrak{g}^{*}}=\mathrm{i} d_{\mathfrak{g}^{*}}$ and the induced map $\bar{\phi}: T_{\theta_{1}}^{*} \mathfrak{g} / \mathfrak{g}^{*} \longrightarrow T_{\theta_{2}}^{*} \mathfrak{g}$ $/ \mathfrak{g}^{*}$ is the identity, i.e., $\phi(x)-x \in \mathfrak{g}^{*}$. Moreover, if $\phi$ is also an isometry, then, $T_{\theta_{1}}^{*} \mathfrak{g}$ and $T_{\theta_{2}}^{*} \mathfrak{g}$ are said to be isometrically equivalent.

Proposition 27. Suppose that $(\mathfrak{g},[\cdot, \cdots, \cdot], \alpha)$ is an n-ary multiplicative Hom-Nambu-Lie superalgebra over a field $\mathbb{K}$ of characteristic not 2 and $\theta_{1}, \theta_{2} \in Z^{1}\left(\mathfrak{g}, \mathfrak{g}^{*}\right)_{\overline{0}}$ satisfying (45). Then, we have

(1) $\left(T_{\theta_{1}}^{*} \mathfrak{g},[\cdot, \cdots, \cdot]_{\theta_{1}}, \alpha^{\prime},\langle,\rangle_{\theta_{1}}\right)$ is equivalent to $\left(T_{\theta_{2}}^{*} \mathfrak{g}\right.$, $\left.[\cdot, \cdots, \cdot]_{\theta_{2}}, \alpha^{\prime},\langle,\rangle_{\theta_{2}}\right)$ if and only if $\theta_{1}-\theta_{2}:=\delta \theta^{\prime} \in \delta C^{0}$ $\left(\mathfrak{g}, \mathfrak{g}^{*}\right)_{\overline{0}}$ and $\theta^{\prime}(x) \alpha=\theta^{\prime}(\alpha(x))$ for all $x \in \mathfrak{g}$. Moreover,

$$
\langle x, y\rangle_{\theta}{ }^{\prime}:=\frac{1}{2}\left(\theta^{\prime}(x)(y)+(-1)^{|x||y|} \theta^{\prime}(y)(x)\right)
$$

becomes a supersymmetric invariant bilinear form on $\mathfrak{g}$ and $\alpha$ is $\langle,\rangle_{\theta}{ }^{\prime}$-symmetric.

(2) $T_{\theta_{1}}^{*} \mathfrak{g}$ is isometrically equivalent to $T_{\theta_{2}}^{*} \mathfrak{g}$ if and only if there is $\theta^{\prime} \in C^{0}\left(\mathfrak{g}, \mathfrak{g}^{*}\right)_{\overline{0}}$ such that $\theta_{1}-\theta_{2}=\delta \theta^{\prime}$ and the bilinear form induced by $\theta^{\prime}$ in (64) vanishes
Proof. (1) Let $\phi: T_{\theta_{1}}^{*} \mathfrak{g} \longrightarrow T_{\theta_{2}}^{*} \mathfrak{g}$ be an isomorphism of $n$-ary multiplicative Hom-Nambu-Lie superalgebras satisfying $\left.\phi\right|_{\mathfrak{g}^{*}}=\mathrm{i} d_{\mathfrak{g}^{*}}$ and $\phi(x)-x \in \mathfrak{g}^{*}, \forall x \in \mathfrak{g}$. Set $\theta^{\prime}(x)=\phi(x)-x$. Then $\theta^{\prime} \in C^{0}\left(\mathfrak{g}, \mathfrak{g}^{*}\right)_{\overline{0}}$ and

$$
\begin{aligned}
0= & \phi\left(\left[x_{1}+f_{1}, \cdots, x_{n}+f_{n}\right]_{\theta_{1}}\right)-\left[\phi\left(x_{1}+f_{1}\right), \cdots, \phi\left(x_{n}+f_{n}\right)\right]_{\theta_{2}} \\
= & \phi\left(\left[x_{1}, \cdots, x_{n}\right]_{\mathfrak{g}}\right)+\theta_{1}\left(x_{1}, \cdots, x_{n}\right) \\
& -\left[x_{1}+\theta^{\prime}\left(x_{1}\right)+f_{1}, \cdots, x_{n}+\theta^{\prime}\left(x_{n}\right)+f_{n}\right]_{\theta_{2}} \\
& +\sum_{i=1}^{n}(-1)^{n-i}(-1)^{\left|x_{i}\right|\left(\left|x_{i+1}\right|+\cdots+\left|x_{n}\right|\right)} \mathrm{a} d^{*}\left(x_{1}, \cdots, \widehat{x}_{i}, \cdots, x_{n}\right) \\
& \cdot f_{i}=\theta^{\prime}\left(\left[x_{1}, \cdots, x_{n}\right]_{\mathfrak{g}}\right)+\theta_{1}\left(x_{1}, \cdots, x_{n}\right)-\theta_{2}\left(x_{1}, \cdots, x_{n}\right) \\
& -\sum_{i=1}^{n}(-1)^{n-i}(-1)^{\left|x_{i}\right|\left(\left|x_{i+1}\right|+\cdots+\left|x_{n}\right|\right)} \mathrm{a} d^{*}\left(x_{1}, \cdots, \widehat{x}_{i}, \cdots, x_{n}\right) \\
& \cdot \theta^{\prime}\left(x_{i}\right)=\theta_{1}\left(x_{1}, \cdots, x_{n}\right)-\theta_{2}\left(x_{1}, \cdots, x_{n}\right)-\delta \theta^{\prime}\left(x_{1}, \cdots, x_{n}\right) .
\end{aligned}
$$

By $\alpha^{\prime} \phi=\phi \alpha^{\prime}$, we may obtain $\theta^{\prime}(x) \alpha=\theta^{\prime}(\alpha(x))$ for all $x \in \mathfrak{g}$.

For the converse, suppose that $\theta^{\prime} \in C^{0}\left(\mathfrak{g}, \mathfrak{g}^{*}\right)_{\overline{0}}$ satisfies $\theta_{1}-\theta_{2}=\delta \theta^{\prime}$ and $\theta^{\prime}(x) \alpha=\theta^{\prime}(\alpha(x))$ for all $x \in \mathfrak{g}$. Let $\phi: T_{\theta_{1}}^{*} \mathfrak{g} \longrightarrow T_{\theta_{2}}^{*} \mathfrak{g}$ be defined by $\phi(x+f)=x+\theta^{\prime}(x)+$ $f$. Then $\left.\phi\right|_{\mathfrak{g}^{*}}=\mathrm{i} d_{\mathfrak{g}^{*}}$ and $\phi(x)-x \in \mathfrak{g}^{*}, \forall x \in \mathfrak{g}$. Moreover, $\alpha^{\prime} \phi$ $=\phi \alpha^{\prime}$. In fact,

$$
\begin{aligned}
& \alpha^{\prime} \phi(x+f)=\alpha\left(x+\theta^{\prime}(x)+f\right)=\alpha(x)+\theta^{\prime}(x) \alpha+f \alpha \\
& \phi \alpha^{\prime}(x+f)=\phi(\alpha(x)+f \alpha)=\alpha(x)+\theta^{\prime}(\alpha(x))+f \alpha .
\end{aligned}
$$

By $\theta^{\prime}(x) \alpha=\theta^{\prime}(\alpha(x))$, one gets $\alpha^{\prime} \phi=\phi \alpha^{\prime}$. Therefore, $\phi$ is an isomorphism of $n$-ary multiplicative Hom-Nambu-Lie superalgebras, that is, $T_{\theta_{1}}^{*} \mathfrak{g}$ is equivalent to $T_{\theta_{2}}^{*} \mathfrak{g}$.

It is clear that $\langle,\rangle_{\theta}{ }^{\prime}$ defined by (64) is supersymmetric. Note that

$$
\begin{aligned}
\langle\mathscr{X} \cdot & y, z\rangle_{\theta}{ }^{\prime}+(-1)^{|\mathscr{X}||y|}\langle y, \mathscr{X} \cdot z\rangle_{\theta}{ }^{\prime} \\
= & \frac{1}{2}\left(\theta^{\prime}(\mathscr{X} \cdot y)(z)+(-1)^{(|\mathscr{X}|+|y|)|z|} \theta^{\prime}(z)(\mathscr{X} \cdot y)\right) \\
& +\frac{1}{2}(-1)^{|X X||y|}\left(\theta^{\prime}(y)(\mathscr{X} \cdot z)+(-1)^{(|\mathscr{X}|+|z|)|y|} \theta^{\prime}(\mathscr{X} \cdot z)(y)\right) \\
= & \frac{1}{2}\left\{\theta_{2}(\mathscr{X}, y)(z)-\theta_{1}(\mathscr{X}, y)(z)+\mathrm{a} d^{*}(\mathscr{X}) \theta^{\prime}(y)(z)\right. \\
& +\sum_{i=1}^{n-1}(-1)^{n-i}(-1)^{\left|x_{i}\right|\left(\left|x_{i+1}\right|+\cdots+\left|x_{n-1}\right|+|y|\right)} \mathrm{a} d^{*}
\end{aligned}
$$




$$
\begin{aligned}
& \left.\cdot\left(x_{1}, \cdots, \widehat{x}_{i}, \cdots, x_{n-1}, y\right) \cdot \theta^{\prime}\left(x_{i}\right)(z)\right\} \\
& -\frac{1}{2}(-1)^{|y||z|} \mathrm{a} d^{*}(\mathscr{X}) \cdot \theta^{\prime}(z)(y)-\frac{1}{2} \mathrm{a} d^{*}(\mathscr{X}) \\
& \cdot \theta^{\prime}(y)(z)+\frac{1}{2}(-1)^{|y||z|}\left\{\theta_{2}(\mathscr{X}, z)(y)\right. \\
& -\theta_{1}(\mathscr{X}, z)(y)+\mathrm{a} d^{*}(X) \theta^{\prime}(z)(y) \\
& +\sum_{i=1}^{n-1}(-1)^{n-i}(-1)^{\left|x_{i}\right|\left(\left|x_{i+1}\right|+\cdots+\left|x_{n-1}\right|+|z|\right)} \mathrm{a} d^{*} \\
& \left.\cdot\left(x_{1}, \cdots, \widehat{x}_{i}, \cdots, x_{n-1}, z\right) \cdot \theta^{\prime}\left(x_{i}\right)(y)\right\}=0,
\end{aligned}
$$

where we make use of $(63)=0$ and $\theta_{1}, \theta_{2}$ satisfying (45). Then, $\langle,\rangle_{\theta}{ }^{\prime}$ is invariant. In addition,

$$
\begin{aligned}
\langle\alpha(x), y\rangle_{\theta}{ }^{\prime} & =\frac{1}{2}\left(\theta^{\prime}(\alpha(x))(y)+(-1)^{|x||y|} \theta^{\prime}(y)(\alpha(x))\right) \\
& =\frac{1}{2}\left(\theta^{\prime}(\alpha(y))(x)+(-1)^{|x||y|} \theta^{\prime}(x)(\alpha(y))\right) \\
& =\langle\alpha(y), x\rangle_{\theta}{ }^{\prime}
\end{aligned}
$$

since $\theta^{\prime}(x) \alpha=\theta^{\prime}(\alpha(x))$ for all $x \in \mathfrak{g}$. That is, $\alpha$ is $\langle x, y\rangle_{\theta}{ }^{\prime}$ -symmetric.

(2) Let the isomorphism $\phi$ be defined as in (1). Then for all $x+f, y+g \in T_{\theta_{1}}^{*} \mathfrak{g}$, we have

$$
\begin{aligned}
\langle\phi(x+f), \phi(y+g)\rangle_{\theta_{2}}= & \left\langle x+\theta^{\prime}(x)+f, y+\theta^{\prime}(y)+g\right\rangle_{\theta_{2}} \\
= & \theta^{\prime}(x)(y)+f(y)+(-1)^{|x||y|} \theta^{\prime}(y)(x) \\
& +(-1)^{|x||y|} g(x)=2\langle x, y\rangle_{\theta}{ }^{\prime} \\
& +\langle x+f, y+g\rangle_{\theta_{1}} .
\end{aligned}
$$

Thus, $\phi$ is an isometry if and only if $\langle,\rangle_{\theta}{ }^{\prime}=0$.

Lemma 28. Let $\left(V,\langle,\rangle_{V}, \alpha\right)$ be a metric $\mathbb{Z}_{2}$-graded vector space of dimension $m$ over an algebraically closed field $\mathbb{K}$ of characteristic not 2 and $\mathfrak{g} \subseteq g l(V)$ be a Lie superalgebra consisting of nilpotent homogeneous endomorphisms of $V$ such that for each $f \in \mathfrak{g}$, the map $f^{+}: V \longrightarrow V$ defined by $\left\langle f^{+}(v), v^{\prime}\right\rangle_{V}=(-1)^{|f||v|}\left\langle v, f\left(v^{\prime}\right)\right\rangle_{V}$ is contained in $\mathfrak{g}$, too. Suppose that $W$ is an isotropic graded subspace of $V$ which is stable under $\mathfrak{g}$ and $\alpha$, i.e., $f(W) \subseteq W$ for all $f \in \mathfrak{g}$ and $\alpha($ $W) \subseteq W$, then $W$ is contained in a maximally isotropic graded subspace $W_{\max }$ of $V$ which is also stable under $\mathfrak{g}$ and $\alpha$, moreover, $\operatorname{dim} W_{\max }=[m / 2]$. If $m$ is even, then $W_{\max }=W_{\max }^{\perp}$. If $m$ is odd, then $W_{\max } \subset W_{\max }^{\perp}$, $\operatorname{dim} W_{\max }^{\perp}$ $-\operatorname{dim} W_{\max }=1$, and $f\left(W_{\max }^{\perp}\right) \subseteq W_{\max }$ for all $f \in \mathfrak{g}$.

Proof. The proof is by induction on $m$. The base step $m=0$ is obviously true. For the inductive step, we consider the following two cases.
Case 1. $W \neq 0$ or there is a nonzero $\mathfrak{g}$-stable vector $v \in V$ (that is, $\mathfrak{g}(v) \subseteq \mathbb{K} v$ ) such that $\langle v, v\rangle_{V}=0$.

Case 2. $W=0$ and every nonzero $\mathfrak{g}$-stable vector $v \in V$ satisfies $\langle v, v\rangle_{V} \neq 0$.

In the first case, $\mathbb{K} v$ is a nonzero isotropic $\mathfrak{g}$-stable graded subspace, and $W^{\perp}$ is also $\mathfrak{g}$-stable since $\left\langle w, f\left(w^{\perp}\right)\right\rangle_{V}=(-1)^{|f||w|}\left\langle f^{+}(w), w^{\perp}\right\rangle_{V}=0$. Now, consider the bilinear form $\langle,\rangle_{V}{ }^{\prime}$ on the factor graded space $V^{\prime}=$ $W^{\perp} / W$ defined by $\left\langle x^{\perp}+W, y^{\perp}+W\right\rangle_{V}{ }^{\prime}:=\left\langle x^{\perp}, y^{\perp}\right\rangle_{V}$, then $V^{\prime}$ is metric. Denote by $\pi$ the canonical projection $W^{\perp} \longrightarrow V^{\prime}$ and define $f^{\prime}: V^{\prime} \longrightarrow V^{\prime}$ by $f^{\prime}\left(\pi\left(w^{\perp}\right)\right)=\pi\left(f\left(w^{\perp}\right)\right)$, then $f^{\prime}$ is well defined since $W$ and $W^{\perp}$ are $\mathfrak{g}$-stable. Let $\mathfrak{g}^{\prime}:=\left\{f^{\prime}\right.$ $\mid f \in \mathfrak{g}\}$. Then, $\mathfrak{g}^{\prime}$ is a Lie superalgebra. For each $f \in \mathfrak{g}$, there is a positive integer $k$ such that $f^{k}=0$, which implies that $\left(f^{\prime}\right)^{k}=0$. Hence, $\mathfrak{g}^{\prime}$ also consists of nilpotent homogeneous endomorphisms of $V^{\prime}$. Note that $\mathfrak{g}^{\prime}$ satisfies the same conditions of $\mathfrak{g}$. In fact, let $x^{\perp}$ and $y^{\perp}$ be two arbitrary elements in $W^{\perp}$. Then, by the definition of $\langle,\rangle_{V}{ }^{\prime}$, we have

$$
\begin{aligned}
& \left\langle\left(f^{\prime}\right)^{+}\left(\pi\left(x^{\perp}\right)\right), \pi\left(y^{\perp}\right)\right\rangle_{V}^{\prime} \\
& =(-1)^{\left|f^{\prime}\right|\left|x^{\perp}\right|}\left\langle\pi\left(x^{\perp}\right), f^{\prime}\left(\pi\left(y^{\perp}\right)\right)\right\rangle_{V}{ }^{\prime} \\
& =(-1)^{|f|\left|x^{\perp}\right|}\left\langle\pi\left(x^{\perp}\right), \pi\left(f\left(y^{\perp}\right)\right)\right\rangle_{V}{ }^{\prime} \\
& =(-1)^{|f|\left|x^{\perp}\right|}\left\langle x^{\perp}, f\left(y^{\perp}\right)\right\rangle_{V}=\left\langle f^{+}\left(x^{\perp}\right), y^{\perp}\right\rangle_{V} \\
& =\left\langle\pi\left(f^{+}\left(x^{\perp}\right)\right), \pi\left(y^{\perp}\right)\right\rangle_{V}{ }^{\prime} \\
& =\left\langle\left(f^{+}\right)^{\prime}\left(\pi\left(x^{\perp}\right)\right), \pi\left(y^{\perp}\right)\right\rangle_{V}^{\prime},
\end{aligned}
$$

for arbitrary $f \in \mathfrak{g}$, which shows that $\left(f^{\prime}\right)^{+}=\left(f^{+}\right)^{\prime} \in \mathfrak{g}^{\prime}$ for all $f \in \mathfrak{g}$.

Since $\operatorname{dim} V^{\prime}=\operatorname{dim} W^{\perp}-\operatorname{dim} W=\operatorname{dim} V-2 \operatorname{dim} W$, we can use the inductive hypothesis to get a maximally isotropic $\mathfrak{g}^{\prime}$-stable subspace $W_{\text {max }}^{\prime}=W_{\max } / W$ in $V^{\prime}$ and $\alpha\left(W_{\text {max }}^{\prime}\right) \subseteq$ $W_{\text {max }}^{\prime}$. Clearly, $\operatorname{dim} W_{\text {max }}^{\prime}=\left[\operatorname{dim} V^{\prime} / 2\right]=[(n-2 \operatorname{dim} W) / 2]$ $=[n / 2]-\operatorname{dim} W$. For all $x^{\perp}, y^{\perp} \in W_{\max }$, the relation $\left\langle x^{\perp}, y^{\perp}\right\rangle_{V}$ $=\left\langle\pi\left(x^{\perp}\right), \pi\left(y^{\perp}\right)\right\rangle_{V}{ }^{\prime}=0$ implies that $W_{\max }$ is isotropic. Note that $\operatorname{dim} W_{\text {max }}=\operatorname{dim} W_{\text {max }}^{\prime}+\operatorname{dim} W=[n / 2]$, then, $W_{\text {max }}$ is maximally isotropic. Moreover, for all $f \in \mathfrak{g}$ and $w^{\perp} \in W_{\max }$, we have $\pi\left(f\left(w^{\perp}\right)\right)=f^{\prime}\left(\pi\left(w^{\perp}\right)\right) \in W_{\text {max }}^{\prime}$, which implies $f\left(w^{\perp}\right)$ $\in W_{\max }$. It follows that $W_{\max }$ is $\mathfrak{g}$-stable and $\alpha\left(W_{\max }\right) \subseteq W_{\max }$. This proves the first assertion of the lemma in this case.

In the second case, by Engel's Theorem of Lie superalgebras, there is a nonzero $\mathfrak{g}$-stable vector $v \in V$ such that $f(v)$ $=0$ for all $f \in \mathfrak{g}$. Clearly, $\mathbb{K} v v$ is a nondegenerate $\mathfrak{g}$-stable graded subspace of $V$, then $V=\mathbb{K} v \widehat{\Theta}(\mathbb{K} v)^{\perp}$ and $(\mathbb{K} v)^{\perp}$ is also $\mathfrak{g}$-stable since $\left\langle f\left((k v)^{\perp}\right), v\right\rangle_{V}=(-1)^{|f||v|}\left\langle(k v)^{\perp}, f^{+}(v)\right\rangle_{V}=$ $(-1)^{|f||v|}\left\langle(k v)^{\perp}, 0\right\rangle_{V}=0, \forall f \in \mathfrak{g}$. Now, if $(\mathbb{K} v)^{\perp}=0$, then $V$ $=\mathbb{K} v$ and $\mathfrak{g}(V)=0$, hence $\mathfrak{g}=0$ and so 0 is the maximally isotropic $\mathfrak{g}$-stable subspace, then the lemma follows. If $(\mathbb{K} v)^{\perp} \neq 0$, then again by Engel's Theorem of Lie 
superalgebras, there is a nonzero $\mathfrak{g}$-stable vector $w \in(\mathbb{K} v)^{\perp}$ $\subseteq V$ such that $f(w)=0$ for all $f \in \mathfrak{g}$. It follows that $\mathfrak{g}$ vanishes on the two-dimensional nondegenerate subspace $\mathbb{K} v \widehat{\cup} \mathbb{K} w$ of $V$. Without loss of generality, we can assume that $\langle v, v\rangle_{V}=$ $1=\langle w, w\rangle_{V}$. Set $c=\langle v, w\rangle_{V}$, then it is easy to check that the nonzero vector $v+\left(-c+\sqrt{c^{2}-1}\right) w$ is isotropic and $\mathfrak{g}$-stable. This contradicts the assumption of Case 2 .

Therefore, the existence of a maximally isotropic $\mathfrak{g}$-stable graded subspace $W_{\max }$ containing $W$ is proved. If $m$ is even, then $\operatorname{dim} W_{\max }=\operatorname{dim} W_{\max }^{\perp}=m / 2 ;$ if $m$ is odd, then $\operatorname{dim} W_{\max }^{\perp}=(m+1) / 2$ and $\operatorname{dim} W_{\max }=(m-1) / 2$. Since $\mathfrak{g}^{\prime}$ is nilpotent, there exists a nonzero $\pi\left(w^{\perp}\right) \in V^{\prime}$ such that $\mathfrak{g}^{\prime}(\pi$ $\left.\left(w^{\perp}\right)\right)=0$. Note that $\operatorname{dim} V^{\prime}=1$, which implies $\mathfrak{g}^{\prime}\left(V^{\prime}\right)=0$, so $\mathfrak{g}\left(W_{\max }^{\perp}\right) \subseteq W_{\max }$.

Theorem 29. Let $\left(\mathfrak{g},[\cdot, \cdots, \cdot], \alpha,\langle,\rangle_{\mathfrak{g}}\right)$ be a nilpotent metric $n$ -ary multiplicative Hom-Nambu-Lie superalgebra of dimension $m$ over an algebraically closed field $\mathbb{K}$ of characteristic not 2. If $J$ is an isotropic Hom-ideal of $\mathfrak{g}$, then $\mathfrak{g}$ contains a maximally Hom-ideal $I$ of dimension $[\mathrm{m} / 2]$ containing $J$. Moreover, if $m$ is even, then $\mathfrak{g}$ is isometric to some $T^{*}$-extension of $\mathfrak{g} / I$. If $m$ is odd, then $I^{\perp}$ is abelian and $\mathfrak{g}$ is isometric to a nondegenerate graded ideal of codimension 1 in some $T^{*}$-extension of $\mathfrak{g} / I$.

Proof. Consider ad $\left(\wedge^{n-1} \mathfrak{g}\right)=\left\{\operatorname{ad} \mathscr{X} \mid \mathscr{X} \in \wedge^{n-1} \mathfrak{g}\right\}$. Then, ad $\left(\wedge^{n-1} \mathfrak{g}\right)$ is a Lie superalgebra. For any $\mathscr{X} \in \wedge^{n-1} \mathfrak{g}$, ad $\mathscr{X}$ is nilpotent since $\mathfrak{g}$ is nilpotent. Then, the following identity

$$
\langle-\mathrm{ad} \mathscr{X}(y), z\rangle_{\mathfrak{g}}=(-1)^{|\mathscr{X}||y|}\langle y, \mathrm{a} d \mathscr{X}(z)\rangle_{\mathfrak{g}}
$$

implies $(\mathrm{a} d \mathscr{X})^{+}:=-\mathrm{a} d \mathscr{X} \in \mathfrak{g}$. By $J$ is an isotropic graded ideal of $\mathfrak{g}$, then, $J$ is an isotropic ad $\left(\wedge^{n-1} \mathfrak{g}\right)$-stable graded subspace and $\alpha(J) \subseteq J$, by Lemma 28 so there is a maximally isotropic a $d\left(\wedge^{n-1} \mathfrak{g}\right)$-stable graded subspace $I$ of $\mathfrak{g}$ containing $J$ such that $\alpha(I) \subseteq I$ and $\operatorname{dim} I=[\mathrm{m} / 2], I$ is also an isotropic graded ideal of $\mathfrak{g}$. Moreover, if $m$ is even, then, $\mathfrak{g}$ is isometric to some $T^{*}$ -extension of $\mathfrak{g} / I$ by Theorem 26 .

If $m$ is odd, then $\operatorname{dim} I^{\perp}-\operatorname{dim} I=1$ and $\operatorname{ad}\left(\wedge^{n-1} \mathfrak{g}\right)\left(I^{\perp}\right)$ $\subseteq I$ by Lemma 28 . Note that

$$
\begin{aligned}
Z(I) & =\left\{x \in \mathfrak{g} \mid[x, I, \mathfrak{g}, \cdots, \mathfrak{g}]_{\mathfrak{g}}=0\right\} \\
& =\left\{x \in \mathfrak{g} \mid\left\langle\mathfrak{g},[x, I, \mathfrak{g}, \cdots, \mathfrak{g}]_{\mathfrak{g}}\right\rangle=0\right\} \\
& =\left\{x \in \mathfrak{g} \mid\left\langle[I, \mathfrak{g}, \cdots, \mathfrak{g}]_{\mathfrak{g}}, x\right\rangle_{\mathfrak{g}}=0\right\} \\
& =[I, \mathfrak{g}, \cdots, \mathfrak{g}]_{\mathfrak{g}}^{\perp}=\left(\mathrm{a} d\left(\wedge^{n-1} \mathfrak{g}\right)(I)\right)^{\perp},
\end{aligned}
$$

which implies that $I^{\perp} \subset\left(\operatorname{ad}\left(\wedge^{n-1} \mathfrak{g}\right)\left(I^{\perp}\right)\right)^{\perp}=Z\left(I^{\perp}\right)$, hence $I^{\perp}$ is abelian.

Take any nonzero element $a \notin \mathfrak{g}$, we define $\alpha^{\prime}$ by

$$
\alpha^{\prime}(x)= \begin{cases}a & \text { if } x=\mathrm{a}, \\ \alpha(x) & \text { if } x \in \mathfrak{g} .\end{cases}
$$

Then, $\mathbb{K} a$ is a 1 -dimensional abelian $n$-ary multiplicative Hom-Nambu-Lie superalgebra. Define a bilinear map $\langle,\rangle_{a}: \mathbb{K} a \times \mathbb{K} a \longrightarrow \mathbb{K}$ by $\langle a, a\rangle_{a}=1$. Then, \langle\rangle$_{a}$ is a nondegenerate supersymmetric invariant bilinear form on $\mathbb{K} a$. Let $\mathfrak{g}^{\prime}=\mathfrak{g} \dot{+} \mathbb{K} a$. Define

$$
\begin{array}{r}
{\left[x_{1}+k_{1} a, \cdots, x_{n}+k_{n} a\right]_{\mathfrak{g}^{\prime}}=\left[x_{1}, \cdots, x_{n}\right]_{\mathfrak{g}},} \\
\left\langle x+k_{1} a, y+k_{2} a\right\rangle_{\mathfrak{g}}{ }^{\prime}=\langle x, y\rangle_{\mathfrak{g}}+\left\langle k_{1} a, k_{2} a\right\rangle_{a} .
\end{array}
$$

Then, $\left(\mathfrak{g}^{\prime},[\cdot, \cdots, \cdot]_{\mathfrak{g}}{ }^{\prime}, \alpha^{\prime},\langle,\rangle_{\mathfrak{g}}{ }^{\prime}\right)$ is a nilpotent metric $n$-ary multiplicative Hom-Nambu-Lie superalgebra since

$$
\begin{aligned}
\left\langle\alpha^{\prime}(x+a), y+a\right\rangle_{\mathfrak{g}}^{\prime} & =\langle\alpha(x)+\alpha(a), y+a\rangle_{\mathfrak{g}}{ }^{\prime} \\
& =\langle\alpha(x), y\rangle_{\mathfrak{g}}+\langle\alpha(a), a\rangle_{a} \\
& =\langle\alpha(y), x\rangle_{\mathfrak{g}}+\langle\alpha(a), a\rangle_{a} \\
& =\left\langle\alpha^{\prime}(y+a), x+a\right\rangle_{\mathfrak{g}}^{\prime}
\end{aligned}
$$

for all $x, y \in \mathfrak{g}$ and $\mathfrak{g}$ is a nondegenerate Hom-ideal of codimension 1 of $\left(\mathfrak{g}^{\prime}, \alpha^{\prime}\right)$. Since $I^{\perp}$ is not isotropic and $\mathbb{K}$ is algebraically closed there exists $z \in I^{\perp}$ and $\langle z, z\rangle_{\mathfrak{g}}=-1$. In addition, we have $\alpha\left(I^{\perp}\right) \subseteq I^{\perp}$ since $\left\langle\alpha^{\prime}\left(v^{\perp}\right), v\right\rangle_{\mathfrak{g}}=\left\langle\alpha(v), v^{\perp}\right\rangle_{\mathfrak{g}}=0$ for $v \in I$ and $v^{\perp} \in I^{\perp}$. Let $b=a+z$ and $I=I+\mathbb{1} \ll b$. Then, $I^{\prime}$ is an $(m+1) / 2$ -dimensional isotropic graded ideal of $\mathfrak{g}^{\prime}$.

In fact, for all $x+k_{1} a+k_{1} z, y+k_{2} a+k_{2} z \in I^{\prime}$,

$$
\begin{aligned}
\langle x+ & \left.k_{1} a+k_{1} z, y+k_{2} a+k_{2} z\right\rangle_{\mathfrak{g}}{ }^{\prime} \\
= & \left\langle x+k_{1} z, y+k_{2} z\right\rangle_{\mathfrak{g}}+\left\langle k_{1} a, k_{2} a\right\rangle_{a} \\
= & \langle x, y\rangle_{\mathfrak{g}}+\left\langle x, k_{2} z\right\rangle_{\mathfrak{g}}+\left\langle k_{1} z, y\right\rangle_{\mathfrak{g}} \\
& +\left\langle k_{1} z, k_{2} z\right\rangle_{\mathfrak{g}}+k_{1} k_{2}=k_{1} k_{2}-k_{1} k_{2}=0 .
\end{aligned}
$$

In light of Theorem 26, we conclude that $\mathfrak{g}^{\prime}$ is isometric to some $T^{*}$-extension of $\mathfrak{g}^{\prime} / I^{\prime}$.

Define $\Phi: \mathfrak{g}^{\prime} \longrightarrow \mathfrak{g} / I, x+\lambda a \mapsto x-\lambda z+I$. Then

$$
\begin{aligned}
& {\left[\Phi\left(x_{1}+\lambda_{1} a\right), \cdots, \Phi\left(x_{n}+\lambda_{n} a\right)\right]_{\mathfrak{g} / I}} \\
& \quad=\left[x_{1}-\lambda_{1} z+I, \cdots, x_{n}-\lambda_{n} z+I\right]_{\mathfrak{g} / I} \\
& \quad=\left[x_{1}, \cdots, x_{n}\right]_{\mathfrak{g}}+I=\Phi\left(\left[x_{1}, \cdots, x_{n}\right]_{\mathfrak{g}}\right) \\
& \quad=\Phi\left(\left[x_{1}+\lambda_{1} a, \cdots, x_{n}+\lambda_{n} a\right]_{\mathfrak{g}}{ }^{\prime}\right),
\end{aligned}
$$

where we use the fact that $I^{\perp}$ is abelian and $\operatorname{ad}\left(\wedge^{n-1} \mathfrak{g}\right)\left(I^{\perp}\right) \subseteq I$. Moreover, $\Phi \alpha=\alpha \Phi$. In fact, for $x+\lambda a \in \mathfrak{g}^{\prime}$, we have

$$
\begin{aligned}
\Phi \alpha(x+\lambda a) & =\Phi(\alpha(x)+\lambda \alpha(a))=\Phi(\alpha(x)+\lambda a) \\
& =\alpha(x)-\lambda z+I=\alpha(x)-\lambda \alpha(z)+I \\
& =\alpha(x-\lambda z+I)=\alpha \Phi(x+\lambda a) .
\end{aligned}
$$


It's clear that $\Phi$ is surjective and $\operatorname{ker} \Phi=I^{\prime}$, so $\mathfrak{g}^{\prime} / I^{\prime} \cong \mathfrak{g} / I$, hence the theorem follows.

Now, we show that there exists an isotropic Hom-ideal in every finite-dimensional metric $n$-ary multiplicative HomNambu-Lie superalgebra and investigate the nilpotent length of $\mathfrak{g} / I$.

Proposition 30. Suppose that $\left(\mathfrak{g},[\cdot, \cdots, \cdot], \alpha,\langle,\rangle_{\mathfrak{g}}\right)$ is a finitedimensional metric n-ary multiplicative Hom-Nambu-Lie superalgebra.

(1) For any graded subspace $V \subseteq \mathfrak{g}, C(V):=\{x \in \mathfrak{g} \mid$ $\left.[x, \mathfrak{g}, \cdots, \mathfrak{g}]_{\mathfrak{g}} \subseteq V\right\}=\left[\mathfrak{g}, \cdots, \mathfrak{g}, V^{\perp}\right]_{\mathfrak{g}}^{\perp}$

(2) $\mathfrak{g}^{m}=C_{m}(\mathfrak{g})^{\perp}$, where $C_{0}(\mathfrak{g})=0, C_{i+1}(\mathfrak{g})=C\left(C_{i}(\mathfrak{g})\right)$

(3) If $\mathfrak{g}$ is nilpotent of length $k$, then $\mathfrak{g}^{i} \subseteq C_{k-i}(\mathfrak{g})$

Proof. The relation

$$
\begin{aligned}
\left\langle C(V),\left[\mathfrak{g}, \cdots, \mathfrak{g}, V^{\perp}\right]_{\mathfrak{g}}\right\rangle_{\mathfrak{g}} & =\left\langle[\mathfrak{g}, \cdots, \mathfrak{g}, C(V)]_{\mathfrak{g}}, V^{\perp}\right\rangle_{\mathfrak{g}} \\
& \subseteq\left\langle V, V^{\perp}\right\rangle_{\mathfrak{g}}=0
\end{aligned}
$$

shows that $C(V) \subseteq\left[\mathfrak{g}, \cdots, \mathfrak{g}, V^{\perp}\right]_{\mathfrak{g}}^{\perp}$. Notice that

$$
\begin{aligned}
\langle & \left.\left\langle\mathfrak{g}, \cdots, \mathfrak{g},\left[\mathfrak{g}, \cdots, \mathfrak{g}, V^{\perp}\right]_{\mathfrak{g}}^{\perp}\right]_{\mathfrak{g}}, V^{\perp}\right\rangle_{\mathfrak{g}} \\
& =\left\langle\left[\mathfrak{g}, \cdots, \mathfrak{g}, V^{\perp}\right]_{\mathfrak{g}}^{\perp},\left[\mathfrak{g}, \cdots, \mathfrak{g}, V^{\perp}\right]_{\mathfrak{g}}\right\rangle_{\mathfrak{g}}=0
\end{aligned}
$$

which implies $\left[\mathfrak{g}, \cdots, \mathfrak{g},\left[\mathfrak{g}, \cdots, \mathfrak{g}, V^{\perp}\right]_{\mathfrak{g}}^{\perp}\right]_{\mathfrak{g}} \subseteq\left(V^{\perp}\right)^{\perp}=V$, i.e., $\left[\mathfrak{g}, \cdots, \mathfrak{g}, V^{\perp}\right]_{\mathfrak{g}}^{\perp} \subseteq C(V)$. Hence, (1) follows.By induction, (2) and (3) can be proved easily.

Theorem 31. Every finite-dimensional nilpotent metric n-ary multiplicative Hom-Nambu-Lie superalgebra $(\mathfrak{g},[\cdot, \cdots, \cdot], \alpha$, $\left.\langle,\rangle_{\mathfrak{g}}\right)$ over an algebraically closed field of characteristic not 2 such that $\alpha(\mathfrak{g})=\mathfrak{g}$ is isometric to (a nondegenerate ideal of codimension 1 of) a $T^{*}$-extension of a nilpotent $n$-ary multiplicative Hom-Nambu-Lie superalgebra whose nilpotent length is at most a half of the nilpotent length of $\mathfrak{g}$.

Proof. Define $J=\sum_{i=0}^{\infty} \mathfrak{g}^{i} \cap C_{i}(\mathfrak{g})$. Since $\mathfrak{g}$ is nilpotent, the sum is finite. Proposition 30 (2) says $\left(\mathfrak{g}^{i}\right)^{\perp}=C_{i}(\mathfrak{g})$, then $\mathfrak{g}^{i} \cap C_{i}(\mathfrak{g})$ is isotropic for all $i \geq 0$. Since

$$
\mathfrak{g}^{i} \supseteq \mathfrak{g}^{j} \supseteq \mathfrak{g}^{j} \cap C_{j}(g), \text { if } i<j
$$

we have

$$
\left(\mathfrak{g}^{j} \cap C_{j}(\mathfrak{g})\right)^{\perp} \supseteq\left(\mathfrak{g}^{i}\right)^{\perp}=C_{i}(\mathfrak{g}) \supseteq C_{i}(\mathfrak{g}) \cap \mathfrak{g}^{i}, \text { if } i<j
$$

It follows that

$$
\left\langle\mathfrak{g}^{i} \cap C_{i}(\mathfrak{g}), \mathfrak{g}^{j} \cap C_{j}(\mathfrak{g})\right\rangle_{\mathfrak{g}}=0, \forall i, j \geq 0
$$

Therefore, $J$ is an isotropic graded ideal of $\mathfrak{g}$. Let $k$ denote the nilpotent length of $\mathfrak{g}$. Using Proposition 30 (3) we can conclude that $\mathfrak{g}^{[(k+1) / 2]} \subseteq C_{[(k+1) / 2]}(\mathfrak{g})$. This implies that $\mathfrak{g}^{[(k+1) / 2]}$ is contained in $J$. By Theorem 29 , there is a maximally isotropic graded ideal $I$ of $\mathfrak{g}$ containing $J \supseteq \mathfrak{g}^{[(k+1) / 2]}$. It means that $\mathfrak{g} / I$ has nilpotent length at most $[(k+1) / 2]$, and the theorem follows.

Remark 32. Most results concerning $T^{*}$-extensions in [20, 24, $26,27]$ are contained in this section as special cases.

\section{Appendix}

Proof of Theorem 33. We now check that $\delta^{m+1} \circ \delta^{m}=0$. In fact, for $f \in C^{m}(\mathfrak{g}, V)$, one gets

$$
\begin{aligned}
& \left(\delta^{m+1} \circ \delta^{m}(f)\right)\left(\mathscr{X}_{1}, \cdots, \mathscr{X}_{m+2}, z\right) \\
& =\sum_{i<j}(-1)^{i}(-1)^{\left|\mathscr{X}_{i}\right|\left(\left|\mathscr{X}_{i+1}\right|+\cdots+\left|\mathscr{X}_{j-1}\right|\right)} \delta^{m} f \\
& \cdot\left(\alpha\left(\mathscr{X}_{1}\right), \cdots, \alpha \widehat{\left(\mathscr{X}_{i}\right)}, \cdots,\left[\mathscr{X}_{i}, \mathscr{X}_{j}\right]_{\alpha}, \cdots, \alpha\left(\mathscr{X}_{m+2}\right), \alpha(z)\right) \\
& +\sum_{i=1}^{m+2}(-1)^{i}(-1)^{\left|\mathscr{X}_{i}\right|\left(\left|\mathscr{X}_{i+1}\right|+\cdots+\left|\mathscr{X}_{m+2}\right|\right)} \delta^{m} f \\
& \left.\cdot\left(\alpha\left(\mathscr{X}_{1}\right), \cdots, \alpha \widehat{\mathscr{X}}_{i}\right), \cdots, \alpha\left(\mathscr{X}_{m+2}\right), \mathscr{X}_{i} \cdot z\right) \\
& +\sum_{i=1}^{m+2}(-1)^{i+1}(-1)^{\left|\mathscr{X}_{i}\right|\left(|f|+\left|\mathscr{X}_{1}\right|+\cdots+\left|\mathscr{X}_{i-1}\right|\right)} \alpha^{m+1}\left(\mathscr{X}_{i}\right) \\
& \cdot \delta^{m} f\left(\mathscr{X}_{1}, \cdots, \widehat{x}_{i}, \cdots, \mathscr{X}_{m+2}, z\right) \\
& +(-1)^{m+1}\left(\delta^{m} f\left(\mathscr{X}_{1}, \cdots, \mathscr{X}_{m+1},\right) \cdot \mathscr{X}_{m+2}\right) \bullet_{\alpha} \alpha^{m+1}(z), \\
& =\sum_{s<t<i<j} a_{i j s t} f\left(\alpha^{2}\left(\mathscr{X}_{1}\right), \cdots, \alpha^{2}\left(\mathscr{X}_{s}\right), \cdots,\left[\alpha\left(\mathscr{X}_{s}\right), \alpha\left(\mathscr{X}_{t}\right)\right]_{\alpha}, \cdots\right. \text {, } \\
& \left.\cdot \widehat{\alpha}^{2\left(\mathscr{X}_{i}\right)}, \cdots, \alpha\left[\mathscr{X}_{i}, \mathscr{X}_{j}\right]_{\alpha}, \cdots, \alpha^{2}\left(\mathscr{X}_{m+2}\right), \alpha^{2}(z)\right) \\
& +\sum_{s<i<t<j} \tilde{a_{i j s t}} f\left(\alpha^{2}\left(\mathscr{X}_{1}\right), \cdots, \alpha^{2}\left(\widehat{X}_{s}\right), \cdots, \alpha^{2}\left(\widehat{X}_{i}\right), \cdots,\right. \\
& \left.\cdot\left[\alpha\left(\mathscr{X}_{s}\right), \alpha\left(\mathscr{X}_{t}\right)\right]_{\alpha}, \cdots, \alpha\left[\mathscr{X}_{i}, \mathscr{X}_{j}\right]_{\alpha}, \cdots, \alpha^{2}\left(\mathscr{X}_{m+2}\right), \alpha^{2}(z)\right) \\
& +\sum_{s<i<j<t} a_{i j s t} f\left(\alpha^{2}\left(\mathscr{X}_{1}\right), \cdots, \alpha^{2}\left(\widehat{X}_{s}\right), \cdots, \alpha^{2}\left(\widehat{X}_{i}\right), \cdots,\right. \\
& \left.\cdot \alpha\left[\mathscr{X}_{i}, \mathscr{X}_{j}\right]_{\alpha}, \cdots,\left[\alpha\left(\mathscr{X}_{s}\right), \alpha\left(\mathscr{X}_{t}\right)\right]_{\alpha}, \cdots, \alpha^{2}\left(\mathscr{X}_{m+2}\right), \alpha^{2}(z)\right) \\
& -\sum_{i<s<t<j} a_{i j s t} f\left(\alpha^{2}\left(\mathscr{X}_{1}\right), \cdots, \alpha^{2}\left(\widehat{X}_{i}\right), \cdots, \alpha^{2}\left(\widehat{X}_{s}\right), \cdots,\right. \\
& \left.\cdot\left[\alpha\left(\mathscr{X}_{s}\right), \alpha\left(\mathscr{X}_{t}\right)\right]_{\alpha}, \cdots, \alpha\left[\mathscr{X}_{i}, \mathscr{X}_{j}\right]_{\alpha}, \cdots, \alpha^{2}\left(\mathscr{X}_{m+2}\right), \alpha^{2}(z)\right)
\end{aligned}
$$




$$
\begin{aligned}
& -\sum_{i<s<j<t} \tilde{a_{i j s t}} f\left(\alpha^{2}\left(\mathscr{X}_{1}\right), \cdots, \alpha^{2}\left(\widehat{X}_{i}\right), \cdots, \alpha^{2}\left(\widehat{X}_{s}\right), \cdots,\right. \\
& \left.\cdot \alpha\left[\mathscr{X}_{i}, \mathscr{X}_{j}\right]_{\alpha}, \cdots,\left[\alpha\left(\mathscr{X}_{s}\right), \alpha\left(\mathscr{X}_{t}\right)\right]_{\alpha}, \cdots, \alpha^{2}\left(\mathscr{X}_{m+2}\right), \alpha^{2}(z)\right) \\
& -\sum_{i<j<s<t} a_{i j s t} f\left(\alpha^{2}\left(\mathscr{X}_{1}\right), \cdots, \alpha^{2}\left(\mathscr{X}_{i}\right), \cdots, \alpha\left[\mathscr{X}_{i}, \mathscr{X}_{j}\right]_{\alpha}, \cdots,\right. \\
& \text { - } \left.\alpha^{2\left(\mathscr{X}_{s}\right)}, \cdots,\left[\alpha\left(\mathscr{X}_{s}\right), \alpha\left(\mathscr{X}_{t}\right)\right]_{\alpha}, \cdots, \alpha^{2}\left(\mathscr{X}_{m+2}\right), \alpha^{2}(z)\right) \\
& +\sum_{k<i<j} \tilde{b_{i j k}} f\left(\alpha^{2}\left(\mathscr{X}_{1}\right), \cdots, \alpha^{2}\left(\widehat{\mathscr{X}}_{k}\right), \cdots, \widehat{\alpha}^{2}\left(\widehat{X}_{i}\right), \cdots,\right. \\
& \left.\cdot\left[\alpha\left(\mathscr{X}_{k}\right),\left[\mathscr{X}_{i}, \mathscr{X}_{j}\right]_{\alpha}\right]_{\alpha}, \cdots, \alpha^{2}\left(\mathscr{X}_{m+2}\right), \alpha^{2}(z)\right) \\
& -\sum_{i<k<j} b_{i j k} f\left(\alpha^{2}\left(\mathscr{X}_{1}\right), \cdots, \alpha^{2\left(\mathscr{X}_{i}\right)}, \cdots, \alpha^{2}\left(\widehat{\mathscr{X}}_{k}\right), \cdots,\right. \\
& \left.\cdot\left[\alpha\left(\mathscr{X}_{k}\right),\left[\mathscr{X}_{i}, \mathscr{X}_{j}\right]_{\alpha}\right]_{\alpha}, \cdots, \alpha^{2}\left(\mathscr{X}_{m+2}\right), \alpha^{2}(z)\right) \\
& -\sum_{i<j<k} \tilde{b_{i k j}} f\left(\alpha^{2}\left(\mathscr{X}_{1}\right), \cdots, \widehat{\alpha}^{2\left(\mathscr{X}_{i}\right)}, \cdots, \alpha^{2}\left(\widehat{X}_{j}\right), \cdots,\right. \\
& \left.\cdot\left[\mathscr{X}_{i}, \mathscr{X}_{j}\right]_{\alpha} \cdot \alpha\left(\mathscr{X}_{k}\right), \cdots, \alpha^{2}\left(\mathscr{X}_{m+2}\right), \alpha^{2}(z)\right) \\
& +\sum_{k<i<j} c_{i j k} f\left(\alpha^{2}\left(\mathscr{X}_{1}\right), \cdots, \alpha^{2}\left(\mathscr{X}_{k}\right), \cdots, \alpha^{2}\left(\widehat{X}_{i}\right), \cdots,\right. \\
& \left.\cdot \alpha\left[\mathscr{X}_{i}, \mathscr{X}_{j}\right]_{\alpha}, \cdots, \alpha^{2}\left(\mathscr{X}_{m+2}\right), \alpha\left(\mathscr{X}_{k}\right) \cdot \alpha(z)\right) \\
& -\sum_{i<k<j} \tilde{c_{i j k}} f\left(\alpha^{2}\left(\mathscr{X}_{1}\right), \cdots, \alpha^{2\left(\mathscr{X}_{i}\right)}, \cdots, \alpha^{2}\left(\widehat{X}_{k}\right), \cdots,\right. \\
& \left.\cdot \alpha\left[\mathscr{X}_{i}, \mathscr{X}_{j}\right]_{\alpha}, \cdots, \alpha^{2}\left(\mathscr{X}_{m+2}\right), \alpha\left(\mathscr{X}_{k}\right) \cdot \alpha(z)\right) \\
& -\sum_{i<j<k} c_{i j k} f\left(\alpha^{2}\left(\mathscr{X}_{1}\right), \cdots, \alpha^{2}\left(\mathscr{X}_{i}\right), \cdots, \alpha\left[\mathscr{X}_{i}, \mathscr{X}_{j}\right]_{\alpha}, \cdots,\right. \\
& \left.\cdot \alpha^{2}\left(\mathscr{X}_{k}\right), \cdots, \alpha^{2}\left(\mathscr{X}_{m+2}\right), \alpha\left(\mathscr{X}_{k}\right) \cdot \alpha(z)\right) \\
& -\sum_{i<j} \tilde{d}_{i j} f\left(\alpha^{2}\left(\mathscr{X}_{1}\right), \cdots, \alpha^{2}\left(\widehat{X}_{i}\right), \cdots, \alpha^{2}\left(\mathscr{X}_{j}\right), \cdots,\right. \\
& \left.\cdot \alpha^{2}\left(\mathscr{X}_{m+2}\right),\left[\mathscr{X}_{i}, \mathscr{X}_{j}\right]_{\alpha} \cdot \alpha(z)\right)+\sum_{k<i<j} e_{i j k} \alpha^{m+1}\left(\mathscr{X}_{k}\right) \\
& \cdot f\left(\alpha\left(\mathscr{X}_{1}\right), \cdots, \alpha \widehat{\left(\mathscr{X}_{k}\right)}, \cdots, \alpha \widehat{\mathscr{X}}_{i}\right), \cdots,\left[\mathscr{X}_{i}, \mathscr{X}_{j}\right]_{\alpha}, \cdots, \\
& \left.\cdot \alpha\left(\mathscr{X}_{m+2}\right), \alpha(z)\right)-\sum_{i<k<j} \tilde{e_{i j k}} \alpha^{m+1}\left(\mathscr{X}_{k}\right) \cdot f\left(\alpha\left(\mathscr{X}_{1}\right), \cdots,\right. \\
& \left.\left.\cdot \alpha \widehat{\mathscr{X}}_{i}\right), \cdots, \alpha \widehat{\left(\mathscr{X}_{k}\right)}, \cdots,\left[\mathscr{X}_{i}, \mathscr{X}_{j}\right]_{\alpha}, \cdots, \alpha\left(\mathscr{X}_{m+2}\right), \alpha(z)\right) \\
& -\sum_{i<j<k} e_{i j k} \alpha^{m+1}\left(\mathscr{X}_{k}\right) \cdot f\left(\alpha\left(\mathscr{X}_{1}\right), \cdots, \alpha \widehat{\mathscr{X}}_{i}\right), \cdots,\left[\mathscr{X}_{i}, \mathscr{X}_{j}\right]_{\alpha} \text {, } \\
& \left.\left.\cdots, \alpha \widehat{\mathscr{X}}_{k}\right), \cdots, \alpha\left(\mathscr{X}_{m+2}\right), \alpha(z)\right)-\sum_{i<j} \tilde{g}_{i j} \alpha^{m}\left(\left[\mathscr{X}_{i}, \mathscr{X}_{j}\right]_{\alpha}\right) \\
& \left.\cdot f\left(\alpha\left(\mathscr{X}_{1}\right), \cdots, \alpha\left(\widehat{\mathscr{X}}_{i}\right), \cdots, \alpha \widehat{(\mathscr{X}}_{j}\right), \cdots, \alpha\left(\mathscr{X}_{m+2}\right), \alpha(z)\right) \\
& +\sum_{i<j \leq m+1} h_{i j}\left(f \left(\alpha\left(\mathscr{X}_{1}\right), \cdots, \alpha\left(\widehat{\mathscr{X}}_{i}\right), \cdots,\left[\mathscr{X}_{i}, \mathscr{X}_{j}\right]_{\alpha}, \cdots,\right.\right. \\
& \left.\left.\cdot \alpha\left(X_{m+1}\right),\right) \cdot \alpha\left(X_{m+2}\right)\right) \bullet \alpha^{m+1}(z)
\end{aligned}
$$$$
+\sum_{k=1}^{m+1}(-1)^{k+m}(-1)^{\left|X_{k}\right|\left(\left|X_{k+1}\right|+\cdots+\left|X_{m+1}\right|\right)}
$$$$
\cdot\left(f\left(\alpha\left(X_{1}\right), \cdots, \alpha\left(\widehat{X}_{k}\right), \cdots, \alpha\left(X_{m+1}\right),\right) \cdot\left[\mathscr{X}_{k}, X_{m+2}\right]_{\alpha}\right) \bullet \alpha^{m+1}(z)
$$$$
+\sum_{s<t<i} c_{s t i} f\left(\alpha^{2}\left(\mathscr{X}_{1}\right), \cdots, \alpha^{2}\left(\mathscr{X}_{s}\right), \cdots,\right.
$$$$
\left.\cdot\left[\alpha\left(\mathscr{X}_{s}\right), \alpha\left(\mathscr{X}_{t}\right)\right]_{\alpha}, \cdots, \alpha^{2}\left(\mathscr{X}_{i}\right), \cdots, \alpha^{2}\left(\mathscr{X}_{m+2}\right), \alpha\left(\mathscr{X}_{i} \cdot z\right)\right)
$$$$
+\sum_{s<i<t} \tilde{c_{s t i}} f\left(\alpha^{2}\left(\mathscr{X}_{1}\right), \cdots, \alpha^{2}\left(\widehat{\mathscr{X}}_{s}\right), \cdots, \alpha^{2}\left(\widehat{\mathscr{X}}_{i}\right), \cdots,\right.
$$$$
\left.\cdot\left[\alpha\left(\mathscr{X}_{s}\right), \alpha\left(\mathscr{X}_{t}\right)\right]_{\alpha}, \cdots, \alpha^{2}\left(\mathscr{X}_{m+2}\right), \alpha\left(\mathscr{X}_{i} \cdot z\right)\right)
$$$$
-\sum_{i<s<t} c_{s t i} f\left(\alpha^{2}\left(\mathscr{X}_{1}\right), \cdots, \alpha^{2}\left(\widehat{\mathscr{X}}_{i}\right), \cdots, \alpha^{2}\left(\widehat{\mathscr{X}}_{s}\right), \cdots,\right.
$$$$
\left.\cdot\left[\alpha\left(\mathscr{X}_{s}\right), \alpha\left(\mathscr{X}_{t}\right)\right]_{\alpha}, \cdots, \alpha^{2}\left(\mathscr{X}_{m+2}\right), \alpha\left(\mathscr{X}_{i} \cdot z\right)\right)
$$$$
+\sum_{k<i} \tilde{d}_{i k} f\left(\alpha^{2}\left(\mathscr{X}_{1}\right), \cdots, \alpha^{2}\left(\widehat{\mathscr{X}}_{k}\right), \cdots, \alpha^{2}\left(\mathscr{X}_{i}\right), \cdots,\right.
$$$$
\left.\cdot \alpha^{2}\left(\mathscr{X}_{m+2}\right), \alpha\left(\mathscr{X}_{k}\right) \cdot\left(\mathscr{X}_{i} \cdot z\right)\right)-\sum_{i<k} d_{i k} f\left(\alpha^{2}\left(\mathscr{X}_{1}\right), \cdots,\right.
$$$$
\left.\cdot \widehat{\alpha}^{2\left(\mathscr{X}_{i}\right)}, \cdots, \alpha^{2}\left(\widehat{X}_{k}\right), \cdots, \alpha^{2}\left(X_{m+2}\right), \alpha\left(\mathscr{X}_{k}\right) \cdot\left(\mathscr{X}_{i} \cdot z\right)\right)
$$$$
+\sum_{k<i} p_{k i} \alpha^{m+1}\left(\mathscr{X}_{k}\right) \cdot f\left(\alpha\left(\mathscr{X}_{1}\right), \cdots, \alpha \widehat{\left(\mathscr{X}_{k}\right)}, \cdots, \widehat{\alpha\left(\mathscr{X}_{i}\right)}, \cdots,\right.
$$$$
\left.\cdot \alpha\left(\mathscr{X}_{m+2}\right), \mathscr{X}_{i} \cdot z\right)-\sum_{i<k} \tilde{p}_{k i} \alpha^{m+1}\left(\mathscr{X}_{k}\right) \cdot f\left(\alpha\left(\mathscr{X}_{1}\right), \cdots,\right.
$$$$
\left.\cdot \widehat{\alpha\left(\mathscr{X}_{i}\right)}, \cdots, \widehat{\alpha\left(\mathscr{X}_{k}\right)}, \cdots, \alpha\left(\mathscr{X}_{m+2}\right), \mathscr{X}_{i} \cdot z\right)+\sum_{i=1}^{m+1}(-1)^{i+m}
$$$$
\cdot(-1)^{\left|X_{i}\right|\left(\left|X_{i+1}\right|+\cdots+\left|X_{m+2}\right|\right) \cdot\left(f\left(\alpha\left(X_{1}\right), \cdots, \alpha\left(X_{i}\right) \wedge, \cdots, \alpha\left(X_{m+1}\right)\right), \cdot \alpha\left(X_{m+2}\right)\right) \bullet_{\alpha} \alpha^{m}\left(X_{i} z\right)}
$$$$
+\left(f\left(\alpha\left(\mathscr{X}_{1}\right), \cdots, \alpha\left(\mathscr{X}_{m}\right),\right) \cdot \alpha\left(\mathscr{X}_{m+1}\right)\right) \bullet_{\alpha} \alpha^{m}\left(\mathscr{X}_{m+2} \cdot z\right)
$$$$
+\sum_{s<t<i} e_{s t i} \alpha^{m+1}\left(\mathscr{X}_{i}\right) \cdot f\left(\alpha\left(\mathscr{X}_{1}\right), \cdots, \alpha \widehat{\left(\mathscr{X}_{s}\right)}, \cdots,\right.
$$$$
\left.\cdot\left[\mathscr{X}_{s}, \mathscr{X}_{t}\right]_{\alpha}, \cdots, \alpha\left(\widehat{\mathscr{X}}_{i}\right), \cdots, \alpha\left(\mathscr{X}_{m+2}\right), \alpha(z)\right)
$$$$
+\sum_{s<i<t} \tilde{e}_{s t i} \alpha^{m+1}\left(\mathscr{X}_{i}\right) \cdot f\left(\alpha\left(\mathscr{X}_{1}\right), \cdots, \alpha\left(\widehat{\mathscr{X}}_{s}\right), \cdots, \widehat{\alpha\left(\mathscr{X}_{i}\right.}\right), \cdots,
$$$$
\left.\cdot\left[\mathscr{X}_{s}, \mathscr{X}_{t}\right]_{\alpha} \cdots, \alpha\left(X_{m+2}\right), \alpha(z)\right)-\sum_{i<s<t} e_{s t i} \alpha^{m+1}\left(X_{i}\right)
$$$$
\cdot f\left(\alpha\left(\mathscr{X}_{1}\right), \cdots, \alpha\left(\widehat{\mathscr{X}}_{i}\right), \cdots, \alpha \widehat{\mathscr{X}}_{s}\right), \cdots,\left[\mathscr{X}_{s}, \mathscr{X}_{t}\right]_{\alpha} \cdots,
$$$$
\left.\cdot \alpha\left(\mathscr{X}_{m+2}\right), \alpha(z)\right)+\sum_{k<i} \tilde{p_{i k}} \alpha^{m+1}\left(\mathscr{X}_{i}\right) \cdot f\left(\alpha\left(\mathscr{X}_{1}\right), \cdots,\right.
$$$$
\left.\cdot \alpha\left(\widehat{X}_{k}\right), \cdots, \alpha\left(\widehat{X}_{i}\right), \cdots, \alpha\left(\mathscr{X}_{m+2}\right), \mathscr{X}_{k} \cdot z\right)
$$$$
-\sum_{i<k} p_{i k} \alpha^{m+1}\left(\mathscr{X}_{i}\right) \cdot f\left(\alpha\left(\mathscr{X}_{1}\right), \cdots, \alpha \widehat{\left(\mathscr{X}_{i}\right)}, \cdots, \alpha \widehat{\left(\mathscr{X}_{k}\right)}, \cdots,\right.
$$$$
\left.\cdot \alpha\left(X_{m+2}\right), \mathscr{X}_{k} \cdot z\right)+\sum_{k<i} \tilde{g_{k i}} \alpha^{m+1}\left(\mathscr{X}_{i}\right) \cdot\left(\alpha^{m}\left(\mathscr{X}_{k}\right)\right.
$$$$
\left.\cdot f\left(\mathscr{X}_{1}, \cdots, \widehat{X}_{k}, \cdots, \widehat{X}_{i}, \cdots, X_{m+2}, z\right)\right)-\sum_{i<k} g_{k i} \alpha^{m+1}\left(\mathscr{X}_{i}\right)
$$$$
\cdot\left(\alpha^{m}\left(\mathscr{X}_{k}\right) \cdot f\left(\mathscr{X}_{1}, \cdots, \widehat{\mathscr{X}}_{i}, \cdots, \widehat{\mathscr{X}}_{k}, \cdots, \mathscr{X}_{m+2}, z\right)\right)
$$$$
-\sum_{i=1}^{m+1}(-1)^{i+m}(-1)^{\left|X_{i}\right|\left(|f|+\left|X_{1}\right|+\cdots+\left|X_{i-1}\right|\right)} \alpha^{m+1}\left(\mathscr{X}_{i}\right)
$$$$
\cdot\left(\left(f\left(\mathscr{X}_{1}, \cdots, \widehat{X}_{i}, \cdots, X_{m+1},\right) \cdot \mathscr{X}_{m+2}\right) \cdot{ }_{\alpha} \alpha^{m}(z)\right)
$$$$
-(-1)^{\left|X_{m+2}\right|\left(|f|+\left|X_{1}\right|+\cdots+\left|X_{m+1}\right|\right)} \alpha^{m+1}\left(\mathscr{X}_{m+2}\right)
$$$$
\cdot\left(\left(f\left(\mathscr{X}_{1}, \cdots, \mathscr{X}_{m}\right) \cdot \mathscr{X}_{m+1}\right) \bullet_{\alpha} \alpha^{m}(z)\right)
$$ 


$$
\begin{aligned}
& -\sum_{s<t \leq m+1} h_{s t}\left(f\left(\alpha\left(\mathscr{X}_{1}\right), \cdots, \alpha \widehat{\alpha \mathscr{X}_{s}}\right), \cdots,\left[\mathscr{X}_{s}, \mathscr{X}_{t}\right]_{\alpha}, \cdots,\right. \\
& \left.\left.\cdot \alpha\left(\mathscr{X}_{m+1}\right),\right) \cdot \alpha\left(\mathscr{X}_{m+2}\right)\right) \bullet_{\alpha} \alpha^{m+1}(z) \\
& -\sum_{i=1}^{n-1} \sum_{k=1}^{m+1}(-1)^{m+k}(-1)^{\left(|f|+\left|X_{1}\right|+\cdots+\left|X_{m+1}\right|\right)\left(\left|X_{m+2}^{1}\right|+\cdots+\left|X_{m+2}^{i-1}\right|\right)} \\
& \cdot(-1)^{\left|X_{k}\right|\left(\left|X_{k+1}\right|+\cdots+\left|X_{m+1}\right|\right)} \cdot\left[\alpha^{m+1}\left(X_{m+2}^{1}\right), \cdots, f\right. \\
& \left.\cdot\left(\alpha\left(\mathscr{X}_{1}\right), \cdots, \alpha \widehat{\mathscr{X}}_{k}\right), \cdots, \alpha\left(\mathscr{X}_{m+1}\right), \mathscr{X}_{k} \cdot \mathscr{X}_{m+2}^{i}\right), \cdots, \\
& \left.\cdot \alpha^{m+1}\left(X_{m+2}^{n-1}\right), \alpha^{m+1}(z)\right]+\sum_{i=1}^{n-1} \sum_{k=1}^{m+1} \\
& \cdot(-1)^{m+k}(-1)^{\left(|f|+\left|X_{1}\right|+\cdots+\left|X_{m+1}\right|\right)\left(\left|X_{m+2}^{1}\right|+\cdots+\left|X_{m+2}^{i-1}\right|\right)} \\
& \cdot(-1)^{\left|X_{k}\right|\left(|f|+\left|\mathscr{X}_{1}\right|+\cdots+\left|X_{k-1}\right|\right)} \cdot\left[\alpha^{m+1}\left(\mathscr{X}_{m+2}^{1}\right), \cdots, \alpha^{m}\left(\mathscr{X}_{k}\right) \cdot f\right. \\
& \left.\cdot\left(\mathscr{X}_{1}, \cdots, \widehat{X}_{k}, \cdots, X_{m+1}, \mathscr{X}_{m+2}^{i}\right), \cdots, \alpha^{m+1}\left(\mathscr{X}_{m+2}^{n-1}\right), \alpha^{m+1}(z)\right] \\
& -\sum_{i=1}^{n-1}(-1)^{\left(|f|+\left|X_{1}\right|+\cdots+\left|X_{m+1}\right|\right)\left(\left|X_{m+2}^{1}\right|+\cdots+\left|X_{m+2}^{i-1}\right|\right)} \\
& \cdot\left[\alpha^{m+1}\left(\mathscr{X}_{m+2}^{1}\right), \cdots,\left(f\left(\mathscr{X}_{1}, \cdots, X_{m},\right) \cdot \mathscr{X}_{m+1}\right) \bullet{ }_{\alpha} \alpha^{m}\right. \\
& \left.\cdot\left(\mathscr{X}_{m+2}^{i}\right), \cdots, \alpha^{m+1}\left(\mathscr{X}_{m+2}^{n-1}\right), \alpha^{m+1}(z)\right] \text {, }
\end{aligned}
$$

where

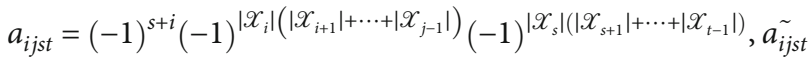

$$
\begin{aligned}
& =(-1)^{\left|X_{i}\right|\left|X_{s}\right|} a_{i j s t} ; \\
& b_{i j k}=(-1)^{i+k}(-1)^{\left|X_{i}\right|\left(\left|X_{i+1}\right|+\cdots+\left|X_{j-1}\right|\right)}(-1)^{\left|X_{k}\right|\left(\left|X_{k+1}\right|+\cdots+\left|X_{j-1}\right|\right)}, \tilde{b_{i j k}} \\
& =(-1)^{\left|X_{i}\right|\left|X_{k}\right|} b_{i j k} ; \\
& c_{i j k}=(-1)^{i+k}(-1)^{\left|\mathscr{X}_{i}\right|\left(\left|X_{i+1}\right|+\cdots+\left|X_{j-1}\right|\right)}(-1)^{\left|X_{k}\right|\left(\left|X_{k+1}\right|+\cdots+\left|X_{m+2}\right|\right)}, \tilde{c_{i j k}} \\
& =(-1)^{\left|X_{i}\right|\left|X_{k}\right|} c_{i j k} \text {; } \\
& d_{i j}=(-1)^{i+j}(-1)^{\mid X_{i}\left(\left|X_{i+1}\right|+\cdots+\left|X_{m+2}\right|\right)}(-1)^{\left|X_{j}\right|\left(\left|X_{j+1}\right|+\cdots+\left|X_{m+2}\right|\right)}, \tilde{d}_{i j} \\
& =(-1)^{\left|\mathscr{X}_{i}\right|\left|\mathscr{X}_{j}\right|} d_{i j} ;
\end{aligned}
$$



$$
\begin{aligned}
& =(-1)^{\left|X_{i}\right|\left|X_{k}\right|} e_{i j k} \text {; } \\
& g_{i j}=(-1)^{i+j+1}(-1)^{\left|X_{i}\right|\left(|f|+\left|X_{1}\right|+\cdots+\left|X_{i-1}\right|\right)}(-1)^{\left|X_{j}\right|\left(|f|+\left|X_{1}\right|+\cdots+\left|X_{j-1}\right|\right)}, \tilde{g}_{i j} \\
& =(-1)^{\left|X_{i}\right|\left|X_{j}\right|} g_{i j} \text {; } \\
& h_{i j}=(-1)^{i+m}(-1)^{\left|X_{i}\right|\left(\left|X_{i+1}\right|+\cdots+\left|X_{j-1}\right|\right)}, \tilde{h_{i j}}=(-1)^{\left|X_{i}\right|\left|X_{j}\right|} h_{i j} \text {; } \\
& p_{k i}=(-1)^{i+k+1}(-1)^{\left|\mathscr{X}_{i}\right|\left(\left|X_{i+1}\right|+\cdots+\left|X_{m+2}\right|\right)}(-1)^{\left|X_{k}\right|\left(|f|+\left|X_{1}\right|+\cdots+\left|X_{k-1}\right|\right)}, \tilde{p_{k i}} \\
& =(-1)^{\left|X_{i}\right|\left|X_{k}\right|} p_{k i} \text {. }
\end{aligned}
$$

It can be verified that the sum of terms labeled with the same letter vanishes. For example, (11) + (12) + (13) + (14) + $(15)=0$, in fact,

$$
\begin{aligned}
& (11)+(12)+(13)+(14)+(15)=\sum_{k=1}^{m+1}(-1)^{k+m}(-1)^{\left|X_{k}\right|\left(\left|X_{k+1}\right|+\cdots+\left|X_{m+1}\right|\right)} \\
& \left.\cdot\left(f\left(\alpha\left(\mathscr{X}_{1}\right), \cdots, \alpha \widehat{\left(\mathscr{X}_{k}\right.}\right), \cdots, \alpha\left(\mathscr{X}_{m+1}\right)\right) \cdot\left[\mathscr{X}_{k}, \mathscr{X}_{m+2}\right]_{\alpha}\right) \bullet \alpha^{m+1}(z) \\
& +\sum_{i=1}^{m+1}(-1)^{i+m}(-1)^{\left|X_{i}\right|\left(\left|X_{i+1}\right|+\cdots+\left|X_{m+2}\right|\right)} \cdot\left(f \left(\alpha\left(X_{1}\right), \cdots,\right.\right. \\
& \left.\left.\cdot \alpha\left(\widehat{\mathscr{X}}_{i}\right), \cdots, \alpha\left(\mathscr{X}_{m+1}\right),\right) \cdot \alpha\left(\mathscr{X}_{m+2}\right)\right) \bullet{ }_{\alpha} \alpha^{m}\left(\mathscr{X}_{i} \cdot z\right) \\
& -\sum_{i=1}^{m+1}(-1)^{i+m}(-1)^{\left|X_{i}\right|\left(|f|+\left|X_{1}\right|+\cdots+\left|X_{i-1}\right|\right)} \cdot \alpha^{m+1}\left(\mathscr{X}_{i}\right) \\
& \cdot\left(\left(f\left(x_{1}, \cdots, \widehat{x}_{i}, \cdots, x_{m+1},\right) \cdot x_{m+2}\right) \cdot{ }_{\alpha} \alpha^{m}(z)\right) \\
& \text { - } \sum_{i=1}^{n-1} \sum_{k=1}^{m+1}(-1)^{m+k}(-1)^{\left(|f|+\left|X_{1}\right|+\cdots+\left|X_{m+1}\right|\right)\left(\left|X_{m+2}^{1}\right|+\cdots+\left|X_{m+2}^{i-1}\right|\right)} \\
& \cdot(-1)^{\left|X_{k}\right|\left(\left|X_{k+1}\right|+\cdots+\left|X_{m+1}\right|\right)} \cdot\left[\alpha^{m+1}\left(\mathscr{X}_{m+2}^{1}\right), \cdots, f\left(\alpha\left(\mathscr{X}_{1}\right), \cdots,\right.\right. \\
& \left.\left.\cdot \alpha\left(\widehat{X}_{k}\right), \cdots, \alpha\left(\mathscr{X}_{m+1}\right), \mathscr{X}_{k} \cdot \mathscr{X}_{m+2}^{i}\right), \cdots, \alpha^{m+1}\left(\mathscr{X}_{m+2}^{n-1}\right), \alpha^{m+1}(z)\right] \\
& +\sum_{i=1}^{n-1} \sum_{k=1}^{m+1}(-1)^{m+k}(-1)^{\left(|f|+\left|X_{1}\right|+\cdots+\left|x_{m+1}\right|\right)\left(\left|X_{m+2}^{1}\right|+\cdots+\left|X_{m+2}^{i-1}\right|\right)} \\
& \cdot(-1)^{\left|X_{k}\right|\left(|f|+\left|X_{1}\right|+\cdots+\left|X_{k-1}\right|\right)} \cdot\left[\alpha^{m+1}\left(\mathscr{X}_{m+2}^{1}\right), \cdots, \alpha^{m}\left(\mathscr{X}_{k}\right) \cdot f\right. \\
& \left.\cdot\left(X_{1}, \cdots, \widehat{X}_{k}, \cdots, X_{m+1}, X_{m+2}^{i}\right), \cdots, \alpha^{m+1}\left(\mathscr{X}_{m+2}^{n-1}\right), \alpha^{m+1}(z)\right]
\end{aligned}
$$

and

$$
\begin{aligned}
& \text { (11) }=\sum_{k=1}^{m+1}(-1)^{k+m}(-1)^{\left|X_{k}\right|\left(\left|X_{k+1}\right|+\cdots+\left|X_{m+1}\right|\right)} \\
& \cdot \sum_{i=1}^{n-1}(-1)^{\left|X_{k}\right|\left(\left|X_{m+2}^{1}\right|+\cdots+\left|X_{m+2}^{i-1}\right|\right)} \\
& \cdot\left(f\left(\alpha\left(\mathscr{X}_{1}\right), \cdots, \alpha\left(\widehat{\mathscr{X}}_{k}\right), \cdots, \alpha\left(\mathscr{X}_{m+1}\right),\right)\right. \\
& \cdot\left(\alpha\left(X_{m+2}^{1}\right) \wedge \cdots \wedge \mathscr{X}_{k} \cdot \mathscr{X}_{m+2}^{i} \wedge \cdots \wedge \alpha\left(\mathscr{X}_{m+2}^{n-1}\right)\right) \bullet{ }_{\alpha} \alpha^{m+1}(z) \\
& =\sum_{k=1}^{m+1}(-1)^{k+m}(-1)^{\left|\mathscr{X}_{k}\right|\left(\left|\mathscr{X}_{k+1}\right|+\cdots+\left|\mathscr{X}_{m+1}\right|\right)} \\
& \cdot \sum_{i=1}^{n-1}(-1)^{\left|X_{k}\right|\left(\left|X_{m+2}^{1}\right|+\cdots+\left|X_{m+2}^{i-1}\right|\right)}
\end{aligned}
$$



$$
\begin{aligned}
& \cdot\left[\alpha^{m+1}\left(\mathscr{X}_{m+2}^{1}\right), \cdots, f\left(\alpha\left(\mathscr{X}_{1}\right), \cdots, \alpha \widehat{\mathscr{X}}_{k}\right), \cdots, \alpha\left(\mathscr{X}_{m+1}\right)\right. \text {, } \\
& \left.\left.\cdot \alpha\left(X_{m+2}^{j}\right)\right), \cdots, \alpha^{m}\left(X_{k} \cdot X_{m+2}^{i}\right), \cdots, \alpha^{m+1}\left(X_{m+2}^{n-1}\right), \alpha^{m+1}(z)\right] \\
& +\sum_{j>i}(-1)^{\left(|f|+\left|X_{1}\right|+\cdots+\left|X_{k} \wedge\right|+\cdots+\left|X_{m+1}\right|\right)\left(\left|X_{m+2}^{1}\right|+\cdots+\left|X_{m+2}^{j-1}\right|+\left|X_{k}\right|\right)}
\end{aligned}
$$




$$
\begin{aligned}
\cdot & {\left[\alpha^{m+1}\left(\mathscr{X}_{m+2}^{1}\right), \cdots, \alpha^{m}\left(\mathscr{X}_{k} \cdot \mathscr{X}_{m+2}^{i}\right), \cdots, f\left(\alpha\left(\mathscr{X}_{1}\right), \cdots\right.\right.} \\
\cdot & \left.\left.\left.\alpha \widehat{\left(\mathscr{X}_{k}\right.}\right), \cdots, \alpha\left(\mathscr{X}_{m+1}\right), \alpha\left(\mathscr{X}_{m+2}^{j}\right)\right), \cdots, \alpha^{m+1}\left(\mathscr{X}_{m+2}^{n-1}\right), \alpha^{m+1}(z)\right] \\
+ & (-1)\left(|f|+\left|\mathscr{X}_{1}\right|+\cdots+\left|\mathscr{X}_{k} \wedge\right|+\cdots+\left|\mathscr{X}_{m+1}\right|\right)\left(\left|\mathscr{X}_{m+2}^{1}\right|+\cdots+\left|\mathscr{X}_{m+2}^{i-1}\right|\right) \\
\cdot & {\left[\alpha^{m+1}\left(\mathscr{X}_{m+2}^{1}\right), \cdots, f\left(\alpha\left(\mathscr{X}_{1}\right), \cdots, \alpha \widehat{\left(\mathscr{X}_{k}\right.}\right), \cdots, \alpha\left(\mathscr{X}_{m+1}\right), \mathscr{X}_{k}\right.} \\
\cdot & \left.\left.\left.\mathscr{X}_{m+2}^{i}\right), \cdots, \alpha^{m+1}\left(\mathscr{X}_{m+2}^{n-1}\right), \alpha^{m+1}(z)\right]\right\} .
\end{aligned}
$$

Moreover, we have

$$
\begin{aligned}
& (\mathrm{l} 3)=-\sum_{i=1}^{m+1}(-1)^{i+m}(-1)^{\left|X_{i}\right|\left(|f|+\left|X_{1}\right|+\cdots+\left|X_{i-1}\right|\right)} \\
& \cdot \sum_{j=1}^{n-1}(-1)^{\left(|f|+\left|X_{1}\right|+\cdots+\left|X_{i} \wedge\right|+\cdots+\left|X_{m+1}\right|\right)\left(\left|X_{m+2}^{1}\right|+\cdots+\left|X_{m+2}^{j-1}\right|\right)} \alpha^{m+1}\left(X_{i}\right) \\
& \cdot\left[\alpha^{m}\left(\mathscr{X}_{m+2}^{1}\right), \cdots, f\left(\mathscr{X}_{1}, \cdots, \widehat{X}_{i}, \cdots, \mathscr{X}_{m+1}, \mathscr{X}_{m+2}^{j}\right), \cdots, \alpha^{m}\right. \\
& \left.\cdot\left(\mathscr{X}_{m+2}^{n-1}\right), \alpha^{m}(z)\right]=-\sum_{i=1}^{m+1}(-1)^{i+m}(-1)^{\left|X_{i}\right|\left(|f|+\left|X_{1}\right|+\cdots+\left|X_{i-1}\right|\right)} \\
& \cdot \sum_{j=1}^{n-1}(-1)^{\left(|f|+\left|X_{1}\right|+\cdots+\left|X_{i} \wedge\right|+\cdots+\left|X_{m+1}\right|\right)\left(\left|X_{m+2}^{1}\right|+\cdots+\left|X_{m+2}^{j-1}\right|\right)} \\
& \cdot\left\{\sum _ { l < j } ( - 1 ) ^ { | X _ { i } | ( | X _ { m + 2 } ^ { 1 } | + \cdots + | X _ { m + 2 } ^ { l - 1 } | ) } \left[\alpha^{m+1}\left(X_{m+2}^{1}\right), \cdots, \alpha^{m}\left(X_{i}\right) \cdot \alpha^{m}\right.\right. \\
& \left(\mathscr{X}_{m+2}^{l}\right), \cdots, \alpha \circ f\left(X_{1}, \cdots, \widehat{X}_{i}, \cdots, X_{m+1}, \mathscr{X}_{m+2}^{j}\right), \cdots, \alpha^{m+1}\left(\mathscr{X}_{m+2}^{n-1}\right) \text {, } \\
& \left.\cdot \alpha^{m+1}(z)\right]+\sum_{l>j}(-1)^{\left|X_{i}\right|\left(|f|+\left|X_{1}\right|+\cdots+\left|X_{i} \wedge\right|+\cdots+\left|X_{m+1}\right|+\left|X_{m+2}^{1}\right|+\cdots+\left|X_{m+2}^{l-1}\right|\right)} \\
& \cdot\left[\alpha^{m+1}\left(X_{m+2}^{1}\right), \cdots, \alpha \circ f\left(X_{1}, \cdots, \widehat{X}_{i}, \cdots, X_{m+1}, \mathscr{X}_{m+2}^{j}\right), \cdots, \alpha^{m}\right. \\
& \left.\cdot\left(\mathscr{X}_{i}\right) \cdot \alpha^{m}\left(\mathscr{X}_{m+2}^{l}\right), \cdots, \alpha^{m+1}\left(\mathscr{X}_{m+2}^{n-1}\right), \alpha^{m+1}(z)\right] \\
& +(-1)^{\left|X_{i}\right|\left(\left|X_{m+2}^{1}\right|+\cdots+\left|X_{m+2}^{j-1}\right|\right)} \cdot\left[\alpha^{m+1}\left(\mathscr{X}_{m+2}^{1}\right), \cdots, \alpha^{m}\left(X_{i}\right) \cdot f\right. \\
& \left.\cdot\left(X_{1}, \cdots, \widehat{X}_{i}, \cdots, X_{m+1}, \mathscr{X}_{m+2}^{j}\right), \cdots, \alpha^{m+1}\left(\mathscr{X}_{m+2}^{n-1}\right), \alpha^{m+1}(z)\right] \\
& +(-1)^{\left|\mathscr{X}_{i}\right|\left(\left|\mathscr{X}_{m+2}\right|+|f|+\left|\mathscr{X}_{1}\right|+\cdots+\left|\mathscr{X}_{i} \wedge\right|+\cdots+\left|\mathscr{X}_{m+1}\right|\right)} \\
& \cdot\left[\alpha^{m+1}\left(X_{m+2}^{1}\right), \cdots, \alpha \circ f\left(X_{1}, \cdots, \widehat{X}_{i}, \cdots, X_{m+1}, X_{m+2}^{j}\right), \cdots, \alpha^{m+1}\right. \\
& \left.\left.\cdot\left(X_{m+2}^{n-1}\right), \alpha^{m}\left(X_{i}\right) \cdot \alpha^{m}(z)\right]\right\} \text {. }
\end{aligned}
$$

Since $(14)+(11 c)=0,(12)+(13 d)=0,(11 a)+(13 b)=0$, $(11 b)+(13 a)=0$, one gets $(11)+(12)+(13)+(14)+(15)=$ 0 . Then $\delta^{m+1} \circ \delta^{m}=0$. Therefore, the proof of Theorem 12 is completed.

\section{Data Availability}

There is no data in my manuscript.

\section{Conflicts of Interest}

The authors declare that they have no conflicts of interest.

\section{Acknowledgments}

This work is supported by the NNSF of China (Nos. 11771069 and 11801066), the NSF of Jilin province (No. 20170101048JC), the project of Jilin province department of education (No. JJKH20180005K), the Fundamental Research Funds for the Central Universities (No. 130014801), the Special Project of Basic Business for Heilongjiang Provincial Education Department (no. 135409317), and the Postdoctoral Scientific Research Developmental Fund of Heilongjiang (no. LBH-Q17175).

\section{References}

[1] Y. Daletskii and V. Kushnirevich, "Inclusion of NambuTakhtajan algebra in formal differential geometry structure," Dopovidi Akademiï Nauk Ukraïni, vol. 4, pp. 12-18, 1996.

[2] N. Cantarini and V. G. Kac, "Classification of simple linearly compact $n$-Lie superalgebras," Communications in Mathematical Physics, vol. 298, no. 3, pp. 833-853, 2010.

[3] V. Abramov, "Matrix 3-Lie superalgebras and Brst supersymmetry," International Journal of Geometric Methods in Modern Physics, vol. 14, no. 11, article 1750160, 2017.

[4] F. Ammar, Z. Ejbehi, and A. Makhlouf, "Cohomology and deformations of Hom-algebras," Journal of Lie Theory, vol. 21, pp. 813-836, 2011.

[5] L. Cai and Y. Sheng, "Purely Hom-Lie bialgebras," Science China Mathematics, vol. 61, no. 9, pp. 1553-1566, 2018.

[6] B. Guan, L. Chen, and B. Sun, "3-ary Hom-Lie superalgebras induced by Hom-Lie superalgebras," Advances in Applied Clifford Algebras, vol. 27, no. 4, pp. 3063-3082, 2017.

[7] J. T. Hartwig, D. Larsson, and S. D. Silvestrov, "Deformations of Lie algebras using $\sigma$-derivations," Journal of Algebra, vol. 295 , no. 2, pp. 314-361, 2006.

[8] D. Larsson and S. D. Silvestrov, "Quasi-Hom-Lie algebras, central extensions and 2-cocycle-like identities," Journal of Algebra, vol. 288, no. 2, pp. 321-344, 2005.

[9] A. Makhlouf and S. Silvestrov, "Notes on 1-parameter formal deformations of Hom-associative and Hom-Lie algebras," Forum Mathematicum, vol. 22, no. 4, pp. 715-739, 2010.

[10] A. Makhlouf and S. Silvestrov, "Hom-algebras and Hom-coalgebras," Journal of Algebra and Its Applications, vol. 9, no. 4, pp. 553-589, 2011.

[11] Y. Sheng, "Representations of Hom-Lie algebras," Algebras and Representation Theory, vol. 15, no. 6, pp. 1081-1098, 2012.

[12] Y. Sheng and C. Bai, "A new approach to Hom-Lie bialgebras," Journal of Algebra, vol. 399, pp. 232-250, 2014.

[13] Y. Sheng and D. Chen, "Hom-Lie 2-algebras," Journal of Algebra, vol. 376, pp. 174-195, 2013.

[14] D. Yau, "Hom-algebras and homology," Journal of Lie Theory, vol. 19, pp. 409-421, 2009.

[15] H. Ataguema, A. Makhlouf, and S. Silvestrov, "Generalization of $n$-ary Nambu algebras and beyond," Journal of Mathematical Physics, vol. 50, no. 8, article 083501, 2009.

[16] F. Ammar, S. Mabrouk, and A. Makhlouf, "Representations and cohomology of $n$-ary multiplicative Hom-Nambu-Lie 
algebras," Journal of Geometry and Physics, vol. 61, no. 10, pp. 1898-1913, 2011.

[17] C. Chevalley and S. Eilenberg, "Cohomology theory of Lie groups and Lie algebras," Transactions of the American Mathematical Society, vol. 63, no. 1, pp. 85-124, 1948.

[18] M. Scheunert and R. B. Zhang, "Cohomology of Lie superalgebras and their generalizations," Journal of Mathematical Physics, vol. 39, no. 9, pp. 5024-5061, 1998.

[19] J. A. Azcárraga and J. M. Izquierdo, " $n$-ary algebras: a review with applications," Journal of Physics A: Mathematical and Theoretical, vol. 284, article 012019, 2011.

[20] Y. Ma and L. Chen, "On the cohomology and extensions of first-class $n$-Lie superalgebras," Communications in Algebra, vol. 42, no. 10, pp. 4578-4599, 2014.

[21] J. M. Casas and X. García-Martínez, "Abelian extensions and crossed modules of Hom-Lie algebras," Journal of Pure and Applied Algebra, vol. 224, no. 3, pp. 987-1008, 2020.

[22] L. Song and R. Tang, "Derivation Hom-Lie 2-algebras and nonabelian extensions of regular Hom-Lie algebras," Journal of Algebra and Its Applications, vol. 17, no. 5, article 1850081, 2018.

[23] L. Song and R. Tang, "Cohomologies, deformations and extensions of $n$-Hom-Lie algebras," Journal of Geometry and Physics, vol. 141, pp. 65-78, 2019.

[24] M. Bordemann, "Nondegenerate invariant bilinear forms on nonassociative algebras," Acta Mathematica Universitatis Comenianae, vol. LXVI, no. 2, pp. 151-201, 1997.

[25] I. Bajo, S. Benayadi, and A. Medina, "Symplectic structures on quadratic Lie algebras," Journal of Algebra, vol. 316, no. 1, pp. 174-188, 2007.

[26] Y. Liu, L. Chen, and Y. Ma, "Hom-Nijienhuis operators and $T^{*}$ -extensions of hom-Lie superalgebras," Linear Algebra and its Applications, vol. 439, no. 7, pp. 2131-2144, 2013.

[27] W. Liu and Z. Zhang, " $T^{*}$-extension of $n$-Lie algebras," Linear and Multilinear Algebra, vol. 61, no. 4, pp. 527-542, 2013. 